\title{
CAPTURE OF IRREGULAR SATELLITES DURING PLANETARY ENCOUNTERS
}

\author{
David Nesvorný, ${ }^{1}$ David Vokrouhlický, ${ }^{1,2}$ and Alessandro Morbidelli ${ }^{3}$ \\ Received 2006 November 23; accepted 2007 January 17
}

\begin{abstract}
More than 90 irregular moons of the Jovian planets have recently been discovered. These moons are an enigmatic part of the solar system inventory. Their origin, which is intimately linked with the origin of the planets themselves, has yet to be adequately explained. Here we investigate the possibility that the irregular moons were captured from the circumsolar planetesimal disk by three-body gravitational reactions. These reactions may have been a frequent occurrence during the time when the outer planets migrated within the planetesimal disk. We propose a new model for the origin of irregular satellites in which these objects are captured from the planetesimal disk during encounters between the outer planets themselves in the model for outer planet migration advocated by Tsiganis and collaborators. Through a series of numerical simulations we show that nearby planetesimals can be deflected into planet-bound orbits during close encounters between planets, and that the overall efficiency of this capture process is large enough to produce populations of observed irregular satellites at Saturn, Uranus, and Neptune. The orbits of captured objects are broadly similar to those of known distant satellites. Jupiter, which typically does not have close encounters with other planets in the model of Tsiganis and coworkers, must have acquired its irregular satellites by a different mechanism. Alternatively, the migration model should be modified to accommodate Jupiter's encounters. Moreover, we find that the original size-frequency distribution of the irregular moons must have significantly evolved by collisions to produce their present populations. Our new model may also provide a plausible explanation for the origin of Neptune's large moon Triton.
\end{abstract}

Key words: planets and satellites: formation

\section{INTRODUCTION}

Historically, it was thought that the planets formed near their current locations. However, starting with the pioneering works of Goldreich \& Tremaine $(1979,1980)$ (for planet-gas disk interactions) in late 1970s and Fernández \& Ip (1984) (for planetplanetesimal disk interactions) in the early 1980 s, it has become clear that the structure of the outer solar system, at least, most likely changed as the planets grew and migrated. As we have broadened our horizons concerning the theory of planet formation, we have significantly increased the size of parameter space that we need to explore. As a result, we have come to the point where we need to use any available constraint on the problem.

One of the most severe constraints on planet formation comes from the distribution of small bodies within the solar system. The asteroid belt, the Kuiper Belt, the Oort cloud, and the Trojan asteroids have provided, and continue to provide, important clues to the origin of the planets (Greenberg et al. 1984; Wetherill 1989, 1992; Safronov 1991; Malhotra 1995; Liou \& Malhotra 1997; Stern \& Colwell 1997; Gomes 1998; Petit et al. 2001; Levison et al. 2001; Kenyon 2002; Luu \& Jewitt 2002; Jewitt \& Luu 2003; Levison \& Morbidelli 2003; Gomes et al. 2004; Bottke et al. 2005; see also a review by Dones et al. [2004] for the Oort cloud and Morbidelli et al. [2005b] for Trojans). However, there is one important population that has been mostly overlooked in our race to understand what small bodies can tell us about planet formation: the satellite systems. Recent studies have shown that the satellites may be able to provide crucial clues that cannot be derived

\footnotetext{
${ }^{1}$ Department of Space Studies, Southwest Research Institute, Boulder, CO 80302, USA.

2 On leave from Institute of Astronomy, Charles University, 18000 Prague 8, Czech Republic.

${ }_{3}^{3}$ Observatoire de la Côte d'Azur, Department Cassiopee, 06304 Nice Cedex 4,
}

from any other source (e.g., Beaugé et al. 2002; Nesvorný et al. 2004; Cuk \& Gladman 2005).

More than 90 irregular moons of the Jovian planets have recently been discovered (Gladman et al. 1998, 2000, 2001b; Sheppard \& Jewitt 2002, 2003; Holman et al. 2004; Kavelaars et al. 2004; Sheppard et al. 2003, 2005, 2006). Unlike regular satellites, the irregular moons revolve around planets at large distances in inclined and eccentric orbits. Their origin has yet to be adequately explained.

The standard model for the formation of the regular satellites is that they formed by accretion in circumplanetary disks (Stevenson 2001; Canup \& Ward 2002, 2006; Mosqueira \& Estrada 2003). This model cannot be applied to the irregular satellites because (1) they are, in general, well separated from the regular satellite systems, making it unlikely that they formed from the same circumplanetary disk, (2) their eccentricities, in general, are too large to have been the result of simple accretion, and most importantly (3) most of them follow retrograde orbits, so they could not have formed in the same disk as the prograde regular satellites.

As a result, the irregular satellites are assumed to have been captured by planets from heliocentric orbits. However, their current orbits cannot result from a purely gravitational, Sun-planetsatellite capture because of time-reversibility arguments (i.e., if there is a path in, there must be a path out because the process can be run backward). Therefore, some sort of stabilization mechanism must have helped satellites to acquire their current orbits.

It has been suggested that irregular satellites were captured from heliocentric orbits (1) via dissipation of their orbital energy by gas drag (Pollack et al. 1979; Cuk \& Burns 2004; Kortenkamp 2005), (2) by collisions between planetesimals (Colombo \& Franklin 1971), or (3) by so-called pull-down capture, in which the planet's gradual growth leads to a capture of objects from the 1:1 mean motion resonance with the planet (Heppenheimer \& Porco 1977). 
All these models have important drawbacks. Model 3 does not include the effects of the circumplanetary gas disk, which should have been present when the planets were growing. Once included, the effects of gas drag become more important than those produced by the planet's growth. In (2), the required orbital change implies a large impactor whose size exceeds the threshold for a catastrophic collision. To stabilize the orbits of the fragments produced by such a collision, ejection speeds $\gtrsim 1 \mathrm{~km} \mathrm{~s}^{-1}$ would have to occur. In contrast, the ejection speeds of large fragments produced by catastrophic collisions do not generally exceed $\sim 100 \mathrm{~m} \mathrm{~s}^{-1}$ (Michel et al. 2001).

Cuk \& Burns (2004) adapted (1) (originally proposed by Pollack et al. 1979) to a realistic structure of the circumplanetary gas disk around a nearly formed Jupiter (e.g., Lubow et al. 1999). They found that the gas drag provides a plausible explanation for the progenitor of the (Himalia) group of prograde irregular satellites at Jupiter. They were, however, unable to explain the origin of the more numerous retrograde satellites at Jupiter, which have orbits that are much larger than the radial extent of the compact circumplanetary gas disk considered by Ćuk \& Burns (2004). It is also not clear whether (1) can possibly apply to Uranus' and Neptune's irregular satellites because of the different characteristics of circumplanetary envelopes of these planets (Pollack et al. 1991, 1996) and their low gas-to-solids ratio.

Spectroscopic studies show a large diversity of colors (ranging from gray to very red) among observed irregular satellites of four outer planets (Cruikshank 1980; Degewij et al. 1980; Dumas et al. 1998; Brown 2000; Sykes et al. 2000; Grav et al. 2003, 2004; Grav \& Holman 2004; Porco et al. 2005). This diversity would be difficult to understand if irregular satellites were captured from a local and dynamically cold population of planetesimals at the planet's heliocentric position, because in such a case the colors should be homogeneous (reflecting the local composition) and there should exist a clear color gradient of irregular satellites with heliocentric distance (i.e., from Jupiter to Neptune). Instead, the observed diversity suggests that the irregular satellites probably sample many different radial locations in the original planetesimal disk and argues against early capture from a dynamically cold disk.

Recent work has pointed out yet another, potentially more serious problem with the capture of irregular satellites by the gasassisted (or other) mechanism at early epochs: These early-formed distant satellites are efficiently removed at later times when large planetesimals (Beaugé et al. 2002) or even planet-sized bodies (see below) sweep through the satellite systems during the migration of the outer planets in the planetesimal disk. Therefore, while different generations of irregular satellites may have existed at different times, many irregular satellites observed today were probably captured relatively late (in a gas-free environment).

Additional support for the late capture of irregular satellites comes from the results of Jewitt \& Sheppard (2005), who inferred from observations that all outer planets may have similar populations of irregular satellites. Such a similarity would not be expected if these moons were captured in the gas-drag model, in which the efficiency of capture is proportional to the amount of circumplanetary gas. Instead, this suggests that satellites were captured by a gravitational mechanism whose efficiency is roughly similar for all outer planets.

Here we determine whether the observed irregular satellites could have been captured from heliocentric orbits during the time when fully formed outer planets gradually eliminate (and migrate within) the planetesimal disk. We propose a new model for capture that we describe in detail in $\S 2$. Our model builds on recent results that show that the outer planets may have formed between
5 and $20 \mathrm{AU}$, experienced a short phase of dynamical instability, and achieved their current locations by interactions with a $\sim 30-40 M_{\bullet}$ planetesimal disk (Tsiganis et al. 2005; Morbidelli et al. 2005b; Gomes et al. 2005). Saturn and the ice giants repeatedly encounter each other in this model (the Nice model) ${ }^{4}$ before they get stabilized by dynamical friction. These repeated encounters between planets remove any distant satellites that may have initially formed around Saturn, Uranus, and Neptune by a gasassisted capture (or via a different mechanism) (regular satellites generally survive because they are more tightly bound to planets; Tsiganis et al. 2005). Our model postulates that a new population of distant satellites formed during the same epoch as a product of planetary encounters.

The framework of the Nice model was recently used by Ćuk \& Gladman (2006) to propose that irregular satellites were captured by gas drag and had their orbits expanded by dynamical instabilities produced by evolving planets. Their model, however, is not fully consistent with the Nice model because Ćuk \& Gladman ignored the effect of planetary encounters. As we discussed above (see also $\S 3.2$ ), the planetary encounters in the Nice model can efficiently remove satellite populations formed in earlier epochs.

In $\S 2$, we describe our numerical model designed to (1) track planets through their migration and mutual close encounters, (2) inject millions of test bodies representing background planetesimals into the encounter zones, and (3) determine the capture efficiency and stability of captured moons. We describe results of this model in $\S 3$. The implications of this work are discussed in $\S 4$.

\section{MODEL}

We propose that the observed irregular satellites could have been captured in permanent orbits around outer planets by gravitational three-body reactions. Two possibilities exist: (1) An exchange reaction, in which a binary system enters the planet's Hill sphere and dissolves, and one component ends in a planetocentric orbit. (2) Third-body assist, in which a massive intruder sweeps through the Hill sphere of a planet and gravitationally stirs up the population of planetesimals temporarily passing through the planet's Hill sphere. While most planetesimals escape from the Hill sphere, some may be stabilized in distant satellite orbits. Model 1 has recently been suggested as a possible way to capture Neptune's satellite Triton (Agnor \& Hamilton 2006).

In (1), the orbital speed of the original binary system must be comparable to the encounter speed (see, e.g., Hut \& Bahcall [1983] for a description of similar processes in astrophysical applications). Since the encounter speeds are generally a few $\mathrm{km} \mathrm{s}^{-1}$, a relatively large total mass of the original binary system is required to assure that the orbital speeds of the binary components are in the correct range. Together, it is assumed in model 1 that fairly large (planet-sized) bodies exist in the disk besides the four outer planets themselves, and that these objects have satellites.

In (2), our new idea is that the satellites could have been captured during the encounters between the giant planets. These encounters had to occur, according to the Nice model of Tsiganis et al. (2005), for the origin of the current architecture of the outer solar system. In this model, the planets formed between 5 and $20 \mathrm{AU}$ and had a transient phase of instability during which they scattered by mutual encounters. Gomes et al. (2005) identified the epoch of planetary encounters in the Nice model with the late heavy bombardment (LHB) $\approx 3.8 \mathrm{Gyr}$ ago when most of the Moon's basins formed (Hartmann et al. 2000). They argued that

\footnotetext{
${ }^{4}$ After Nice Observatory in France where this model was developed.
} 
TABLE 1

The Number of Captured Objects in Our 14 Successful Migration Jobs

\begin{tabular}{|c|c|c|c|c|c|c|c|}
\hline \multirow[b]{2}{*}{$\begin{array}{l}\text { Јов ID } \\
\text { (1) }\end{array}$} & \multirow[b]{2}{*}{$\begin{array}{c}\text { Migration Class } \\
\text { (2) }\end{array}$} & \multirow{2}{*}{$\begin{array}{c}\text { Crossing Time } \\
\text { (Myr) } \\
\text { (3) }\end{array}$} & \multirow[b]{2}{*}{$\begin{array}{c}\text { Total EnCOUNTERS } \\
\text { (4) }\end{array}$} & \multicolumn{4}{|c|}{ Number of Captured Satellites } \\
\hline & & & & $\begin{array}{l}\text { Jupiter } \\
\text { (5) }\end{array}$ & $\begin{array}{l}\text { Saturn } \\
\text { (6) }\end{array}$ & $\begin{array}{l}\text { Uranus } \\
\text { (7) }\end{array}$ & $\begin{array}{l}\text { Neptune } \\
\quad(8)\end{array}$ \\
\hline 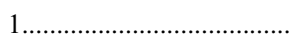 & MA & 1.22 & 221 & 0 & 1 & 736 & 378 \\
\hline 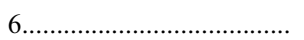 & MA & 0.68 & 221 & 0 & 14 & 139 & 2517 \\
\hline 7.1. & $\mathrm{DE}$ & 5.70 & 943 & 0 & 166 & 416 & 1172 \\
\hline 8 & $\mathrm{DE}$ & 3.11 & 595 & 0 & 1 & 430 & 1552 \\
\hline 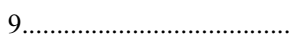 & $\mathrm{DE}$ & 1.29 & 389 & 341 & 437 & 1798 & 219 \\
\hline 11 & MA & 1.07 & 173 & 0 & 35 & 289 & 503 \\
\hline 12 & MA & 0.13 & 55 & 0 & 0 & 427 & 1413 \\
\hline 14 & $\mathrm{DE}$ & 1.59 & 158 & 0 & 51 & 269 & 482 \\
\hline 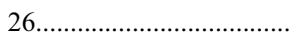 & DE & 1.10 & 313 & 0 & 191 & 548 & 1956 \\
\hline 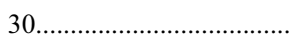 & $\mathrm{MA} / \mathrm{DE}$ & 2.48 & 478 & 0 & 64 & 411 & 69 \\
\hline 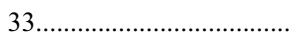 & MA & 0.49 & 122 & 0 & 103 & 1420 & 109 \\
\hline 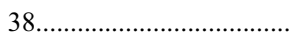 & $\mathrm{MA} / \mathrm{DE}$ & 5.35 & 1033 & 0 & 0 & 79 & 96 \\
\hline 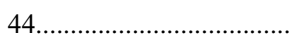 & MA & 2.31 & 224 & 0 & 0 & 159 & 416 \\
\hline 47 & $\mathrm{DE}$ & 2.79 & 443 & 0 & 18 & 568 & 1368 \\
\hline 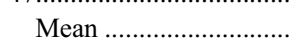 & $\ldots$ & 2.09 & 383 & $\ldots$ & 77.2 & 549 & 937 \\
\hline
\end{tabular}

Notes.-Col. (1): Job's seed value. Col. (2): Migration class (DE: direct emplacement; MA: Malhotra-class migration [see text for a definition of these terms]). Col. (3): Crossing time, defined as the length of the time interval between the first and last planetary encounters. Col. (4): Total number of encounters between planets. Cols. (5-8): Number of stable satellites captured at planets.

the dynamically excited orbits of the outer planets caused a severe depletion of the planetesimal disks at 20-35 AU and that a fraction of these planetesimals evolved into the inner solar system, producing the LHB. To study (2), we use the LHB variant of the Nice model taken from Gomes et al. (2005). In times after the LHB, the planetary orbits become gradually less eccentric due to the effects of dynamical friction from the planetesimal disk and continue to slowly migrate toward their current radial distances in a progressively more and more depleted planetesimal disk.

The population of satellites produced by planetary encounters at the time of the LHB would be long-lived but could be modified during the late migration phase if massive (planet-sized) planetesimals existed in the disk (Beaugé et al. 2002). It is possible that such objects were indeed present in the disk, because it is unlikely that the four giant planets would have been the only large objects to have accreted in this region. Therefore, the populations of irregular satellites observed at the outer planets today may be a complex product of several processes.

Here we focus our work on determining the efficiency of captures during encounters among four outer planets. We conducted the following set of numerical experiments.

In the first step, we used the results of Gomes et al. (2005) to record the orbits of the outer planets and the state of the planetesimal disk at time $t_{0}$. We selected $t_{0}$ so that the state of the system represents the situation shortly before the orbits of Jupiter and Saturn migrate through the 2:1 mean motion resonance, become dynamically excited, and initiate the epoch of planetary encounters. We used one of the published simulations of Gomes et al. (2005) and $t_{0}=868$ Myr.

In the original simulation of Gomes et al. (2005), the pre-LHB planetesimal disk was represented by several hundred objects. We cloned each object several times so that the resulting disk is represented by 6868 objects. The cloning was done as a small perturbation of positions and velocities of the original particles. The masses of the clones were adjusted so that the total mass of the disk and its distribution across the disk were preserved. In total, we created 50 distinct initial states of the disk. Below we identify these states by the seed value we used to initialize the random number generator. The planetesimal disk at $t_{0}$ was located be- tween 21 and $35 \mathrm{AU}$. The positions and velocities of four outer planets (Jupiter to Neptune) at $t_{0}=868 \mathrm{Myr}$ were preserved. The starting semimajor axis of Jupiter, Saturn, and the two ice giants at $t_{0}$ were $5.4,8.4,12.3$, and $18.0 \mathrm{AU}$.

In the second step, we numerically tracked the orbits of planets and planetesimals for at least $130 \mathrm{Myr}$. We used a symplectic integrator known as SyMBA (Duncan et al. 1998). SyMBA is a highly efficient symplectic $N$-body integrator similar to the Wisdom-Holman map (Wisdom \& Holman 1991), which is able to handle close encounters between massive bodies (Skeel \& Biesiadecki 1994). In the code, planets gravitationally interact with each other and also act on other bodies in the simulation. The planetesimals do not interact with each other but affect the orbits of planets. In total, we conducted 50 numerical integration with individual runs that started with slightly different disk states (see above) and produced different migration outcomes. The results of these simulations are described in $\S 3.1$.

The simulations were used to create lists of encounters between planets. Specifically, we recorded every planetary encounter for which the separation of planets became smaller than $R_{\mathrm{H}, 1}+R_{\mathrm{H}, 2}$, where $R_{\mathrm{H}, 1}$ and $R_{\mathrm{H}, 2}$ are the Hill radii of the two planets having the encounter. For each encounter, we recorded the heliocentric positions and velocities of four planets and of all planetesimals. These data were used in the third step.

In the third step, we used only those simulations of step 2 in which the migrating planets at $t_{0}+130 \mathrm{Myr}$ resembled the current outer solar system architecture. We adopted a somewhat loose measure to define this condition. Specifically, we required that none of the planets was ejected from the solar system and that all four planets ended near their current semimajor axis locations with low eccentricities and low inclinations. We avoided a more strict selection of successful runs because we wanted to determine the capture efficiency for a variety of distinct (but still plausible) migration scenarios, rather than limiting the study to a few bestfit cases. We denote the successful runs by their seed values and list them in Table 1.

The information about the sequence of encounters in each successful migration run was used to model the capture in bound orbits about planets. The planetesimal disk was represented by 
several thousand objects in step 2, which was insufficient to detect satellite captures directly. We used the following procedure to increase the statistics. First, we tracked the orbits of planets and planetesimals backward in time from the exact moment of the encounter $\left(t_{\mathrm{enc}}\right)$ to $t=t_{\mathrm{enc}}-\Delta t$, where $\Delta t$ was chosen so that the two approaching planets were separated by more than $5 \mathrm{AU}$ at $t_{\mathrm{enc}}-\Delta t$. With this condition, $\Delta t$ is typically between 10 and $30 \mathrm{yr}$. We used the standard Bulirsch-Stoer method for this numerical integration and in the following steps (Press et al. 1992). We opted for this integration method because it is more flexible than SyMBA for the experiments described here, in which we frequently stop and start integrations, deal with short integration intervals, and track millions of test particles. In this and all following steps the disk planetesimals were assumed to be massless test bodies At $t=t_{\text {enc }}-\Delta t$, we binned semimajor axis $a$, eccentricity $e$, and inclination $i$ of planetesimal orbits in three dimensions. An example of the orbital distribution of planetesimals at $t=t_{\text {enc }}-\Delta t$ is shown in $\S 3.2$.

This detailed information about the orbital distribution of planetesimals before each encounter was used to generate a large number, typically $3 \times 10^{6}$, of disk particles whose orbit density in $a, e$, and $i$ respected the original distribution. The perihelion and nodal longitudes of particles were drawn randomly between $0^{\circ}$ and $360^{\circ}$. The values of mean longitudes were tuned so that disk particles evolve into a sphere with a 3 AU radius around the location of the encounter when planets meet at $t=t_{\text {enc }}$. The above assumptions are legitimate because the disk has not yet developed any important resonant structures at this stage. Our selection criteria typically mean that the generated particles represent $10^{-3}$ to $5 \times 10^{-3}$ of the whole planetesimal disk between 5 and $35 \mathrm{AU}$.

In the next step, the orbits of planets and disk particles were numerically integrated forward in time through the planetary encounter for $2 \Delta t$. At the end of these simulations, orbits of all test bodies were analyzed to determine whether or not they were bound to a planet. The bound orbits were selected for additional numerical integrations that were used to separate long-lived orbits from the unstable ones. Only the captured objects that survived on bound orbits in the time interval up to the subsequent encounter were used.

The subsequent encounter was modeled by exactly the same procedure as described above, except that in addition to the $3 \times$ $10^{6}$ disk particles that we injected into the 3 AU radius sphere around the encounter we also tracked the orbits of planetary satellites produced in all previous encounters. This procedure was iterated over a sequence of encounters. All satellites captured in previous encounters were propagated through all subsequent planetary encounters. This method allowed us to remove the satellites that became destabilized by encounters and identify those that remained on stable orbits after the last encounter. The code allowed us to account for switching reactions in which some satellites may be initially captured by one planet but are handed over to another planet during the following encounters.

Finally, we tracked the orbits of all satellites remaining after the last encounter to determine their stability. We used a fourthorder symplectic integrator in mixed variables (Wisdom \& Holman 1991) for these integrations. Only the long-lived satellites (stable at least for $1 \mathrm{Myr}$ ) were taken into account for the final statistics. We used the above numerical integrations to determine the mean values of $a, e$, and $i$ of all captured objects. These values can be directly compared with the mean values that were reported for the real irregular satellites by Nesvorný et al. (2003). ${ }^{5}$

\footnotetext{
${ }^{5}$ For satellites discovered after 2003, we used the mean elements available at http://ssd.jpl.nasa.gov/?sat_elem.
}

The final state of the satellite swarm at each planet was analyzed and compared with the observed irregular satellites. Specifically, we compared (1) the distributions of semimajor axes, eccentricities, and inclinations, and (2) the number of distant satellites produced at each outer planet. We also analyzed the orbital histories of captured objects to determine (3) their initial distribution in the disk, (4) the exact mechanism of capture, and (5) the orbital evolution of each captured object in the planet's Hill sphere.

\section{RESULTS}

\subsection{Selected Migration Jobs}

Only 14 of our 50 migration runs produced planetary orbits at $t_{0}+130 \mathrm{Myr}$ that were comparable to those of outer planets in our solar system. We list these runs in Table 1. In the remaining cases either at least one of the planets was ejected or Jupiter and Saturn never reached the 2:1 resonance within the integration time span. Apparently, the exact timing of the LHB event is sensitive to the method we used to clone the original disk particle orbits. We do not consider the unsuccessful migration cases in the following.

The 14 successful migration cases can be divided into two classes depending on how exactly the outer planets reached their final positions. In the first class, which we denote as the Malhotra (MA; Malhotra 1995; Hahn \& Malhotra 1999) class, Neptune is scattered to 22-25 AU and reaches its final orbit by slowly migrating in the planetesimal disk over a large radial distance (typically exceeding 5 AU). In the second class, which we denote as the direct emplacement (DE) class, Neptune is placed at its current orbital distance (i.e., $\approx 30 \mathrm{AU}$ ) by a close encounter with Uranus. Neptune's initially eccentric orbit is then circularized by interactions with the planetesimal disk. Tsiganis et al. (2005) favored the DE class over the MA class because the MA-class evolution typically produces orbits of Saturn and Uranus that have much lower eccentricities than the current ones.

Out of our 14 successful runs, six fall into the MA class, six fall into the DE class, and two (runs 30 and 38 in Table 1) represent an intermediate case between $\mathrm{MA}$ and $\mathrm{DE}$ migration types. Figure 1 shows examples of one MA-class evolution (run 44) and one DE-class evolution (run 47). In both cases, the ice giants switched their original positions so that the originally more distant planet ended on a smaller, Uranus-like orbit. The same occurred in all our successful simulations except runs 7 and 8 . The orbital switch between Uranus and Neptune is therefore typical in the Nice model. Hereafter, independent of its original location we identify Neptune as the planet ending near 30 AU.

In run 44, a series of encounters between Uranus and Neptune eventually placed these planets at $\approx 17$ and $\approx 21$ AU, respectively, where their orbits decoupled (about 21 Myr after the start of the simulation). Over the next $100 \mathrm{Myr}$, the ice giants migrated in the planetesimal disk toward their current orbital locations. The migration became slower with time, which is typical for the case of so-called damped migration that occurs in low-mass planetesimal disks (Gomes et al. 2004). In total, Uranus and Neptune migrated by 2 and $7 \mathrm{AU}$, respectively, within the integration time span.

The evolution in the run 47 simulation was different. In that case, Uranus and Neptune interacted more strongly and landed at $\approx 18$ and $\approx 31 \mathrm{AU}$, respectively. Their radial migration for $t>$ 20 Myr was minimal because the planetesimal disk was already severely depleted at $t \approx 20$ Myr. Neptune eventually ended up with a slightly larger orbit than was strictly required. This small difference, however, is irrelevant here. Several other successful 

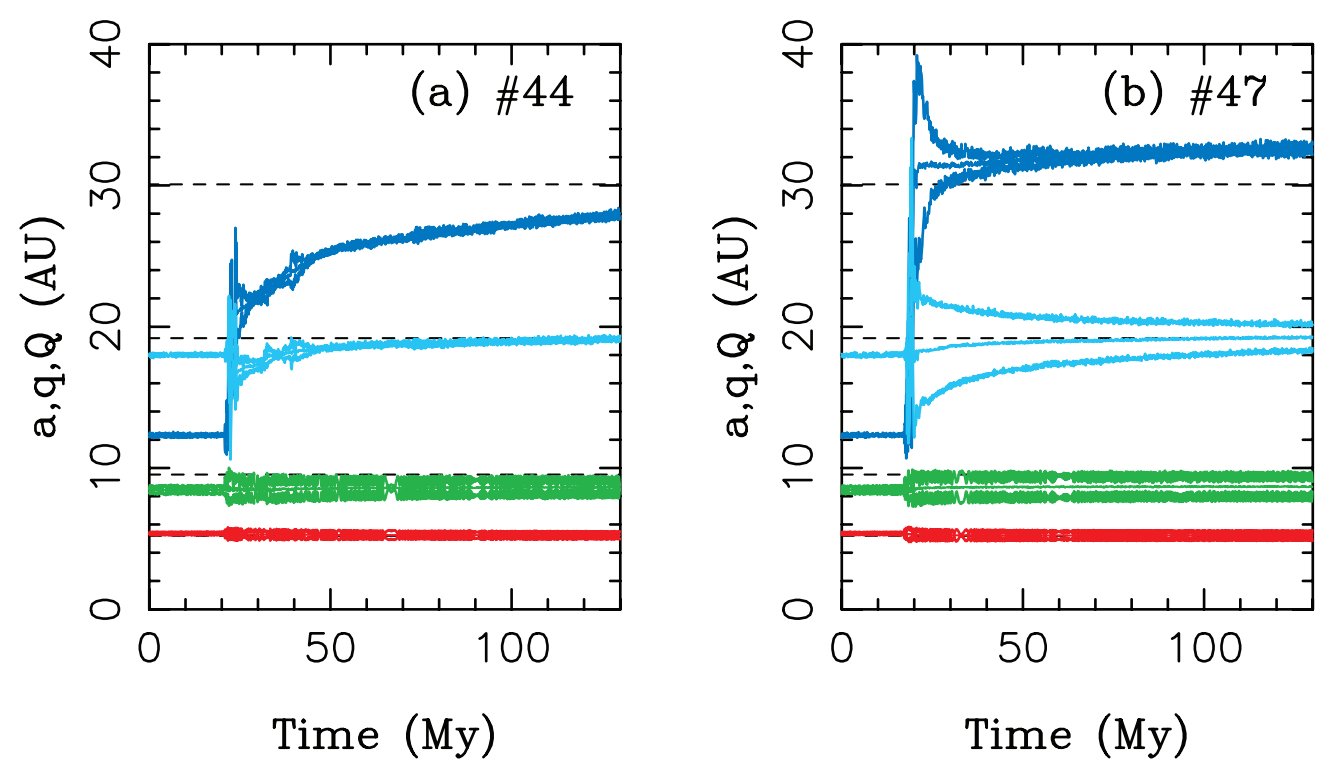

FIG. 1.-Two examples of our migration results, $(a)$ number 44 (MC type of migration) and $(b)$ number 47 (DE type). The solid lines denote the semimajor axis, perihelion distance, and aphelion distances for Jupiter (red), Saturn (green), Uranus (light blue), and Neptune (blue). The dashed lines denote the present values of planetary semimajor axes. Jupiter and Saturn pass through their mutual $2: 1$ resonance about 20 and 18 Myr after the start of the integration in $(a)$ and $(b)$, respectively. The orbits of all planets get dynamically excited following the resonance crossing. In both $(a)$ and $(b)$, Uranus and Neptune switch their radial locations. Their orbits decouple, and dynamical friction from the planetesimal disk then acts to decrease their orbital eccentricities and inclinations. In $(a)$, Uranus and Neptune end up migrating toward their current radial distances. In $(b)$, Uranus and Neptune are placed into their near-final orbits by scattering each other in an encounter.

simulations (runs $1,7,8,11,30$, and 38) produced Neptune's current orbit more precisely.

Figure 2 shows the encounter distances and speeds of approaching planets corresponding to our migration run 47 . In total, the code registered 37 encounters between Saturn and Neptune and 406 encounters between Uranus and Neptune. Most encounters involving Saturn occurred early and during a narrow time window $(\approx 70 \mathrm{kyr})$. They were characterized by $<1.5 \mathrm{~km} \mathrm{~s}^{-1}$ speeds. The speeds of encounters between Uranus and Neptune showed an interesting pattern. They increased during the initial encounters (as expected, because the orbits got dynamically excited) and oscillated between 0.5 and $3 \mathrm{~km} \mathrm{~s}^{-1}$ during the subsequent $\approx 1.5 \mathrm{Myr}$. The time spacing between individual encounters typically varied between 100 and $10,000 \mathrm{yr}$, except for a few intervals with longer delays. The last four encounters between Uranus and Neptune happened after a delay of almost $800 \mathrm{kyr}$.

Figure 3 shows projections of the orbit density binned into $(a, e)$ and $(a, i)$ planes for the last encounter in Figure 2. The last encounter between Uranus and Neptune in this run occurred at $t=20,612,880 \mathrm{yr}$ after $t_{0}$. This planetary encounter had $V_{\infty}=$ $1.317 \mathrm{~km} \mathrm{~s}^{-1}$ and $q=1.145 \mathrm{AU}$, where $V_{\infty}$ is the relative encounter speed of Uranus and Neptune "at infinity" and $q$ is the minimal distance between planets. The encounter happened near radial distance $22.5 \mathrm{AU}$, where the mean eccentricity and inclination of planetesimals were 0.25 and $15^{\circ}$, respectively. This shows that any possible captures during the late phase of planetary encounters must happen from an already excited planetesimal disk. This result is typical for all our migration runs.

There exists a large variability among our different successful migration simulations in the number of planetary encounters, their time spacing, and their duration. The total number of planetary encounters varies from 55 in job 12 to 1033 in job 38 (Table 1). The mean number of encounters in our 14 successful migration simulations is 383 . Most encounters happened between Uranus and Neptune (345 on average). The ones involving Saturn usually happened relatively early in the sequence of encounters
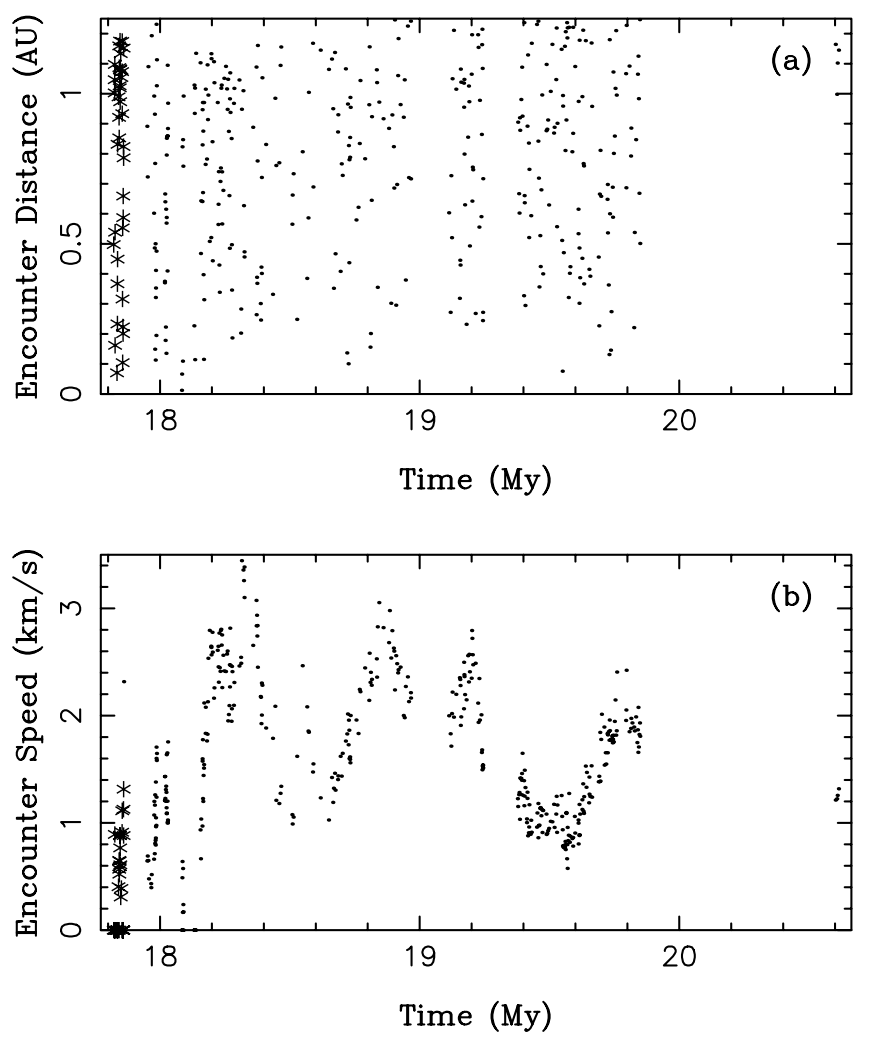

FIG. 2.- Minimum encounter distances and speeds (at infinity) of approaching planets in simulation 47 (corresponding to Fig. 1b). The asterisks show values for encounters between Saturn and Neptune. The dots show encounters between Uranus and Neptune. The encounter speeds between planets were typically $<3 \mathrm{~km} \mathrm{~s}^{-1}$. 

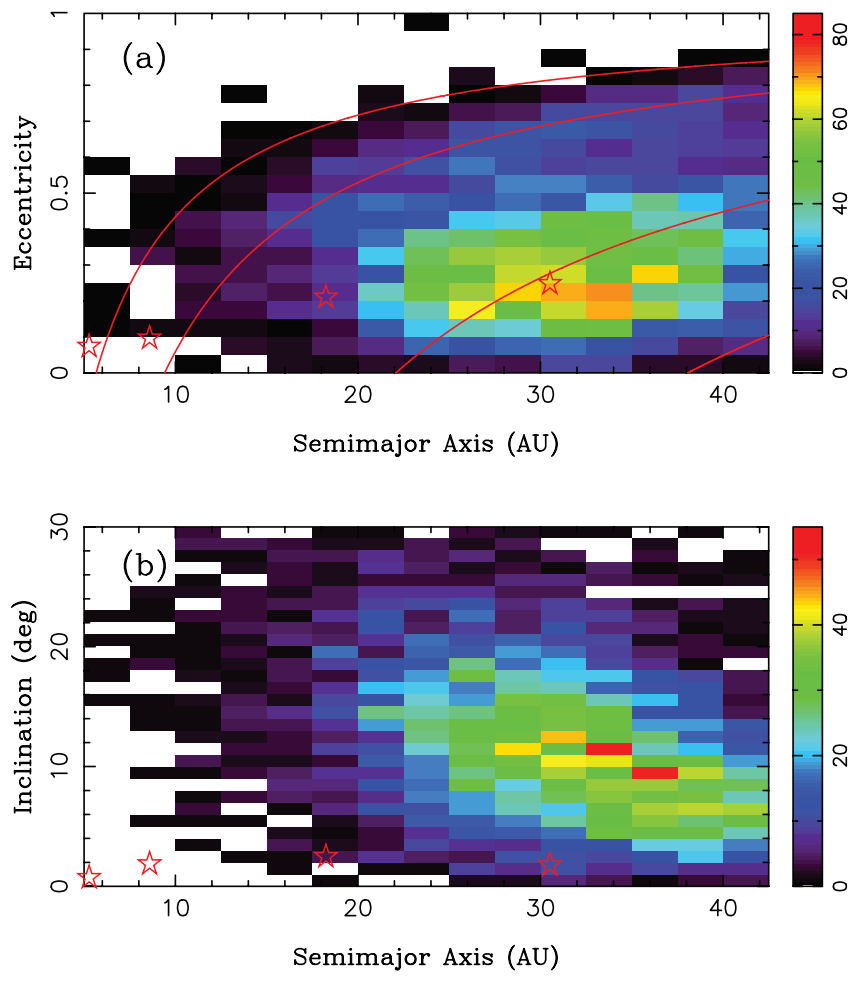

FIG. 3.-Binned orbit density of disk planetesimals at the time of the last encounter between Uranus and Neptune in Fig. 2: $(a)$ projection on the $(a, e)$ plane, and $(b)$ projection on the $(a, i)$ plane. The region between 20 and $40 \mathrm{AU}$ is characterized by the highest orbit density of planetesimals. The orbits of planets are denoted by stars. The solid lines in $(a)$ denote the values of orbit elements above which the orbits are planet crossing. The encounter between Uranus and Neptune happened near radial distance $22.5 \mathrm{AU}$. The mean eccentricity and inclination of planetesimals at that location are 0.25 and $15^{\circ}$, respectively.

and numbered a few to a few dozen. ${ }^{6}$ Jupiter had an encounter with another planet only in jobs 9 (16 encounters with Neptune) and 38 (six encounters with Neptune).

The number of encounters is clearly correlated with the total duration of the time interval during which the planets stay on crossing orbits. The longest durations, 5.7 and 5.4 Myr, were registered for jobs 7 and 38, respectively. Job 12 shows both the smallest number of encounters (55) and shortest duration (0.12 Myr).

The number of encounters is also correlated with the migration class. The average number of encounters in the MA class is only 169 , while it is 473 in the DE class. Moreover, very few encounters with Saturn happen in the MA class $(<5$, except for job 33, with 18 Saturn's encounters) while many of Saturn's encounters happen in the DE class (58 on average). This shows that the DE class is characterized by a much richer history of planetary encounters than the MA class. Consequently, there are more opportunities to capture satellites in the DE class than there are in the MA class.

\subsection{Survival of Satellites Captured Prior to the LHB Epoch}

Tsiganis at al. (2005) and Gomes et al. (2005) found that those satellites that formed before the LHB event do not survive multiple planet encounters. That result was based on a very limited number of tests so that the overall survival efficiency could not have been precisely estimated ( $\mathrm{H}$. Levison 2006, private communication). The main difficulty with estimating the survival rate is

\footnotetext{
${ }^{6}$ Except for jobs 9 and 38, for which the numbers of Saturn's encounters were 198 and 108 , respectively.
}

that it largely varies among different migration runs and depends sensitively on the (assumed) orbits of hypothetical primordial satellites. A detailed analysis of the survival rate is beyond the scope of this work.

We have tried to roughly estimate the survival rate by (1) testing the survivability of primordial satellites placed at different planets on orbits with $e=0$ and $i=0^{\circ}$ or $180^{\circ}$ (their $a$ values were taken between 0.01 and $0.35 \mathrm{AU}$ ), and (2) by analyzing the survivability of distant satellites that were captured during the first encounter between planets. Item 2 is a good proxy for the survival rate of primordial satellites if these originally had moderate $e$ and $i$. Indeed, the disk planetesimals that were captured during the first planet encounter have moderate $e$ and $i$ values. These satellites may be removed or severely depleted by the following planetary encounters, thus approximately mimicking the possible behavior of the pre-LHB-captured objects. Results of our tests mentioned in (1) would be more relevant in the case that the primordial population of distant satellites has had its orbits circularized prior to the LHB by, e.g., the effects of the gas drag (see, e.g., Ćuk \& Burns 2004).

Our results indicate that the primordial populations of satellites started at Uranus and Neptune with $e=0$ and $i=0^{\circ}$ or $180^{\circ}$ are reduced by a factor of $\sim 10-10^{4}$, with the exact value in this range depending on the number of planetary encounters in a specific migration simulation. The migration runs with a large number of planetary encounters tend to produce very low survival rates.

Most surviving satellites have $a \lesssim 0.05$ AU. The ones starting with $a>0.05$ AU become severely depleted and scattered by planetary encounters to orbits with moderate $e$ and inclination values around $0^{\circ}$ or $180^{\circ}$. The orbits started with values of $i$ between $0^{\circ}$ and $180^{\circ}$ tend to have lower survival rates than the ones started with $i=0^{\circ}$ or $i=180^{\circ}$. Taken together, these tests show that any primordial mechanism of capture would have to be very efficient to compensate for the satellite removal during the LHB and explain the population of irregular satellites known today.

Test 2 confirmed these conclusions. About 300-600 satellites became captured around one of the ice giants as a result of the first encounter in the most successful migration jobs. The semimajor axis values of these satellites varied between 0.02 and 0.2 AU. None of these bodies typically survived on a planetbound orbit. We therefore conclude that the survival rate of a primordial population with moderate $e$ and $i$ values is $<2 \times 10^{-3}$. These experiments make us believe that it is unlikely that the origin of the currently known irregular satellites around Uranus and Neptune can date back to pre-LHB epochs.

Conversely, the pre-LHB-captured satellites at Jupiter and Saturn show relatively high survival rates because the gas giants do not generally participate in many planetary encounters. This is especially true for Jupiter. Therefore, the capture of many known irregular satellites of Jupiter (and Saturn) may date back to the preLHB epoch. The existence of secular resonances of several irregular satellites at Jupiter and Saturn (see Beaugé \& Nesvorný 2007 for a recent review) can be viewed as evidence supporting this option. These resonant orbits are difficult to explain by mechanisms active after the LHB. We discuss this issue in more detail in $\S 4$.

As a final comment in this section we note that our tests described above confirm the results of Tsiganis et al. (2005), who found that the orbits of regular satellites of the outer planets suffer only minor perturbations during the planetary encounters. This is because the regular satellites are much more tightly bound to the planets than the irregular satellites, and extremely close planetary encounters are required to destabilize them. Such encounters do not happen in the Nice model. 


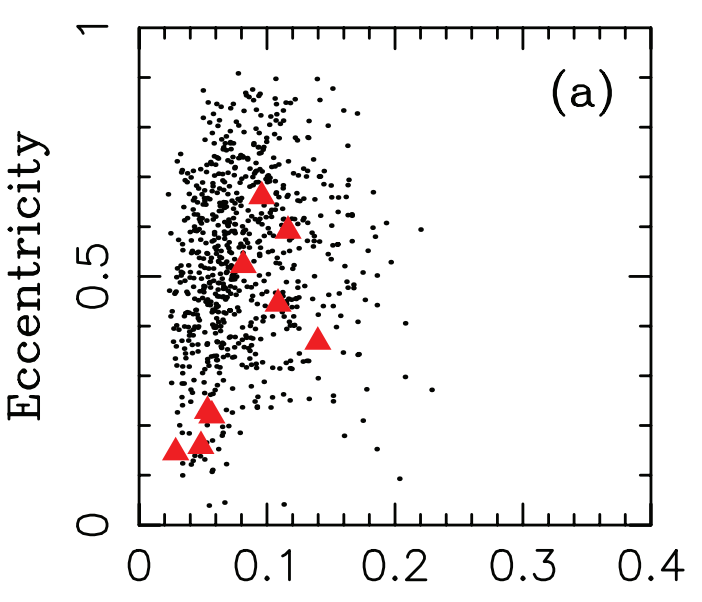

\section{Semimajor Axis (AU)}

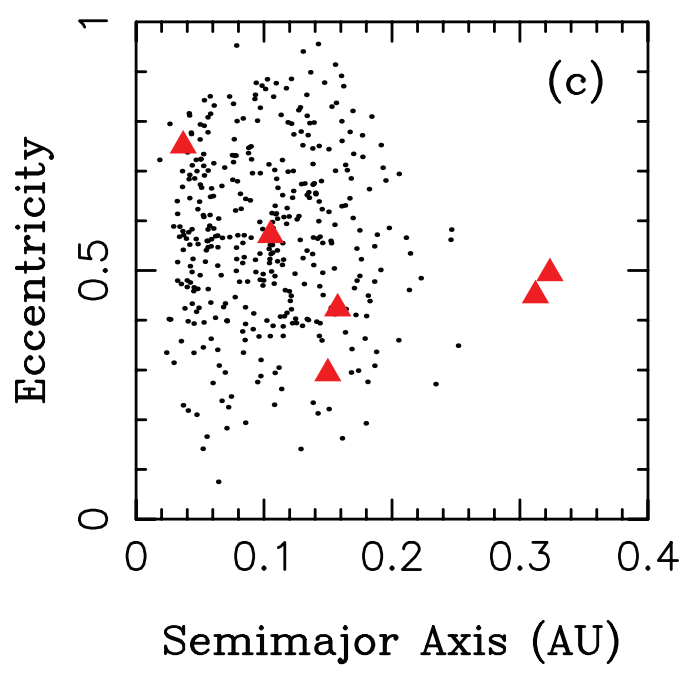

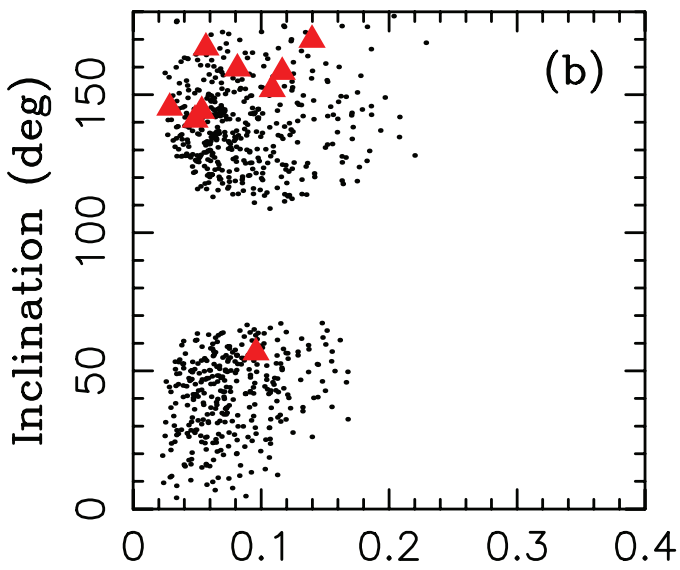

Semimajor Axis (AU)

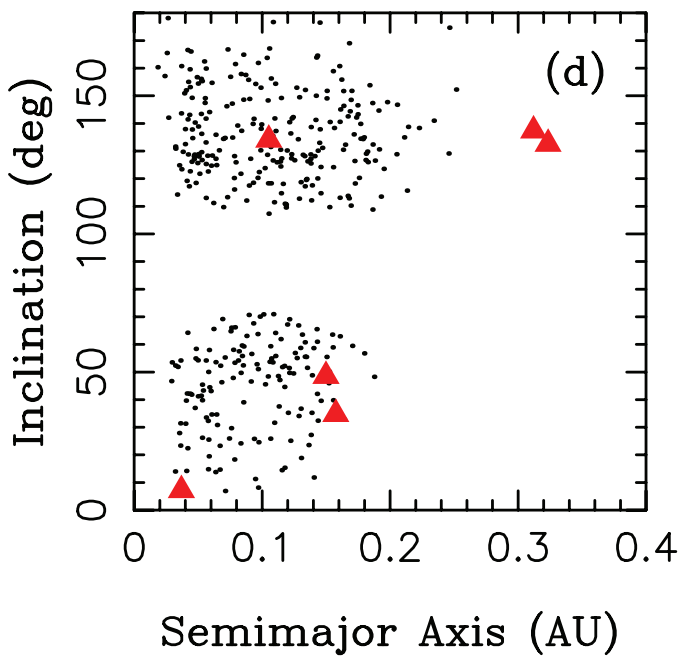

FIG. 4.-Comparison between the orbits of objects captured in job 1 (dots) and those of the known irregular satellites (triangles). $(a, b)$ Satellites of Uranus. $(c, d)$ Satellites of Neptune. In total, 736 and 378 stable satellites were captured in job 1 at Uranus and Neptune, respectively.

\subsection{Orbits of Captured Satellites}

Figures 4 and 5 compare the orbit distributions of captured objects in our jobs 1 (MA class) and 47 (DE class) with those of the observed irregular satellites at Uranus and Neptune. The osculating orbital elements of real irregular satellites were obtained from Horizons. ${ }^{7}$ The elements shown in the figure are the mean orbital elements obtained by averaging $a, e$, and $i$ as described in $\S 2$. In total, planetary encounters in job 1 produced 736 satellites at Uranus and 378 satellites at Neptune (out of $3 \times 10^{6}$ test bodies injected into the $3 \mathrm{AU}$ sphere around each encounter). Job 47 produced 568 satellites at Uranus and 1368 satellites at Neptune. The captured orbits cover a wide range of $a, e$, and $i$.

The agreement between orbits of captured and real satellites for Uranus is good (Figs. $4 a, 4 b, 5 a$, and $5 b$ ). Indeed, the range of $a, e$, and $i$ of captured objects is remarkably similar to that of the real satellites. We note only several small discrepancies that may or may not be important. For example, the group of known retrograde satellites at Uranus may show a tighter inclination range than that of the captured objects. This is true in general for all of our jobs. In fact, the inclination distribution of captured objects

\footnotetext{
${ }^{7}$ See http://ssd.jpl.nasa.gov/?horizons.
}

in our model is similar to $\sin i$, except for $i \sim 90^{\circ}$, where the Kozai resonance produces dynamical instability (Kozai 1962; Carruba et al. 2002; Nesvorný et al. 2003). The inclination distribution proportional to $\sin i$ is expected in our model because the satellites are captured from a dynamically excited disk (see Fig. 3 and $\S 3.4)$.

A more striking difference between the model and observed orbital distributions is that eight out of nine known irregular satellites at Uranus have retrograde orbits while our simulations produce a more symmetric mix of prograde and retrograde orbits. One possible explanation for this discrepancy is that the observed populations may have been shaped by mutual collisions between prograde and retrograde satellites. In the process, the largest irregular moon of Uranus, $\approx 190 \mathrm{~km}$ diameter Sycorax moving on a retrograde orbit, may have eliminated many smaller satellites moving on prograde orbits (see Nesvorný et al. 2003). We do not account for collisions in the model described here.

For Neptune (Figs. $4 c, 4 d, 5 c$, and $5 d$ ), the agreement is very good except possibly for two retrograde satellites, S/2002 N4 and $\mathrm{S} / 2003 \mathrm{~N} 1$, that have very large values of $a(\approx 0.32 \mathrm{AU})$. None of the captured orbits in job 1 shows $a>0.26 \mathrm{AU}$. Conversely, job 47 produced several orbits like those of S/2002 N4 and S/2003 N1. This result is general: the MA-class jobs such as job 1 do not 


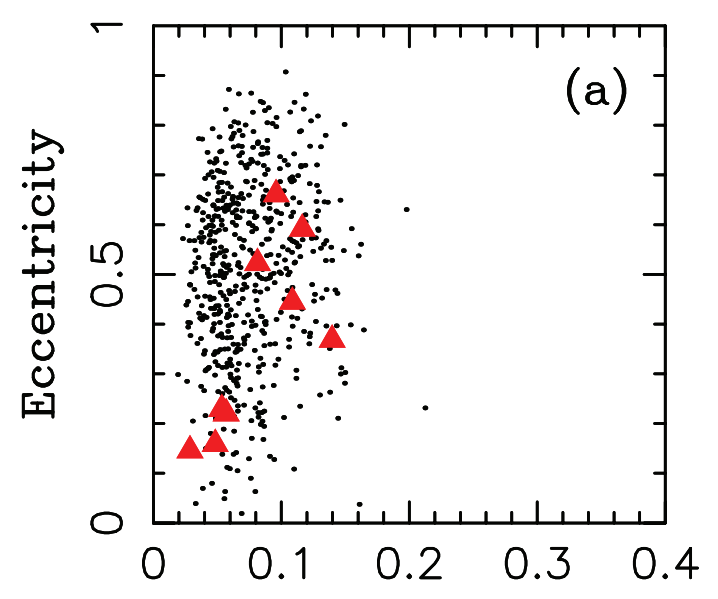

\section{Semimajor Axis (AU)}

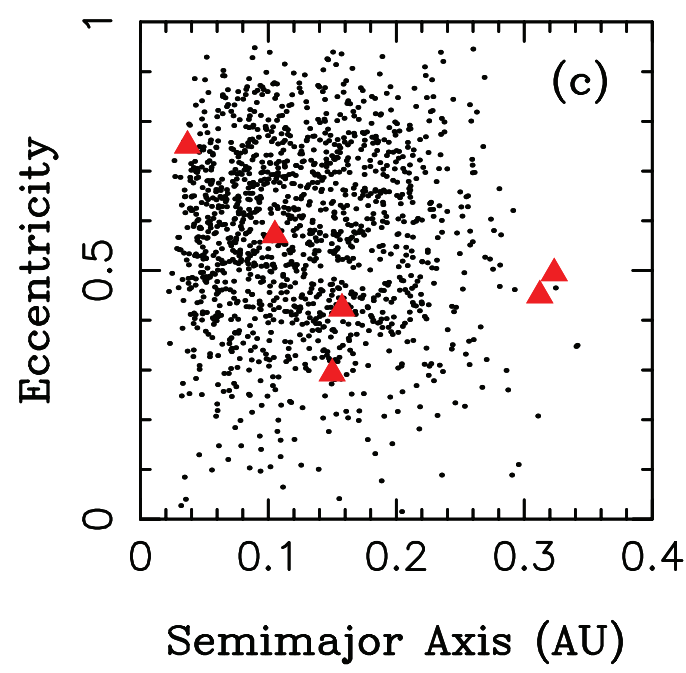

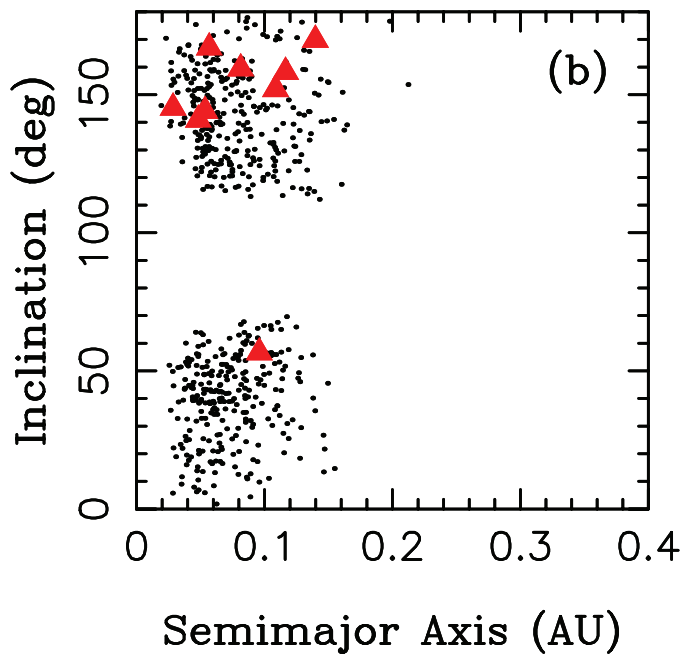

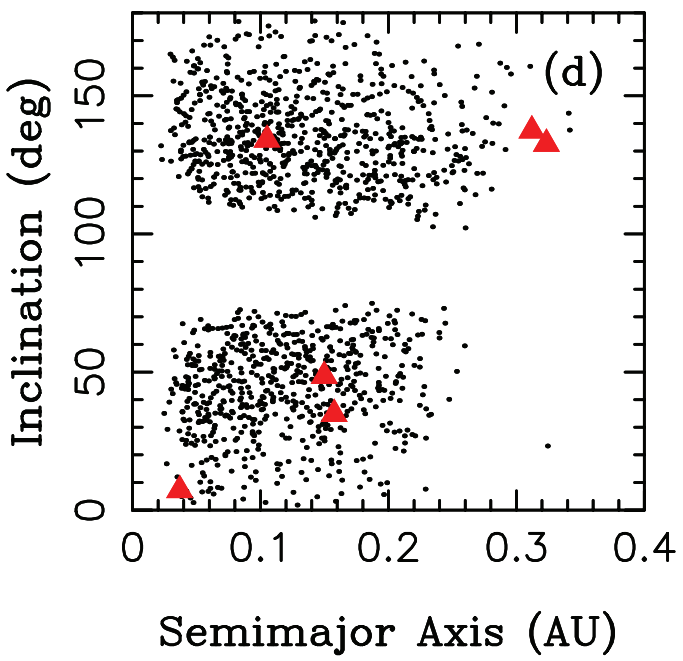

FIG. 5.-Comparison between the orbits of objects captured in job 47 (dots) and those of the known irregular satellites (triangles). $(a, b)$ Satellites of Uranus. $(c, d)$ Satellites of Neptune. In total, 568 and 1368 stable satellites were captured in job 1 at Uranus and Neptune, respectively.

produce orbits with large $a$, while the DE-class jobs such as job 47 do produce them. We explain this behavior below.

The outer limit of the semimajor axis distribution of distant planetary satellites is dictated by the evection resonance (a resonance between the apsidal precession of the satellite's orbit and the orbital motion of its parent planet around the Sun; see, e.g., Kaula \& Yoder 1976; Touma \& Wisdom 1998), which causes strong instabilities beyond $a \sim 0.5 R_{\mathrm{H}}$ (Nesvorný et al. 2003), where $R_{\mathrm{H}}$ is the Hill radius of a planet. The present Hill radius of Neptune is $\approx 0.78$ AU. As Neptune's orbits expanded during migration its Hill sphere grew toward its current value, thus allowing the satellites to be captured with larger and larger $a$.

In the MA class, the last planetary encounter of Neptune typically occurred at $\approx 20$ AU (see Fig. 1 ), where Neptune's $R_{\mathrm{H}} \approx$ 0.52 AU. Therefore, Neptune's satellites produced by planetary encounters in the MA class cannot in principle have $a>0.26 \mathrm{AU}$ because these orbits were unstable in the past. ${ }^{8}$ This result also constrains any mechanism of satellite capture, which would precede Neptune's migration, and points toward yet another fun-

\footnotetext{
${ }^{8}$ Dynamical instabilities produced by the evection resonance act much faster $(<1000 \mathrm{yr}$ timescale $)$ than the timescale for the expansion of Neptune's orbit shown in Fig. 1.
}

damental difficulty with early captures of Neptune's irregular satellites (such as those produced by gas drag). Conversely, the stable satellite orbits captured and scattered by late planetary encounters in the DE class may have significantly larger $a$ values because Neptune's Hill sphere has already expanded when these encounters occurred. This result favors the DE class over the MA class. Alternatively, satellites S/2002 N4 and S/2003 N1 could have been captured or scattered to their current orbits by a different event after Neptune had already reached $\approx 30$ AU (e.g., by a very late capture of Triton).

Figure 6 shows the orbits of captured objects at Saturn in jobs 7 and 47 . We opted to show the results of job 7 here instead of those of job 1 because only one object have been captured at Saturn in job 1. Conversely, jobs 7 and 47 (both DE class) show higher capture efficiency, with 166 and 18 satellites produced at Saturn, respectively. This allowed us to compare the model and real orbit distributions.

The distributions of model and real orbits in Figure 6. are similar, showing essentially the same range of $a, e$, and $i$. This is a remarkable result. It shows that the orbits of known irregular satellites at Saturn, including that of Phoebe, are naturally produced by captures during planetary encounters. This result is general for all of our migration jobs that produce enough satellites at Saturn 

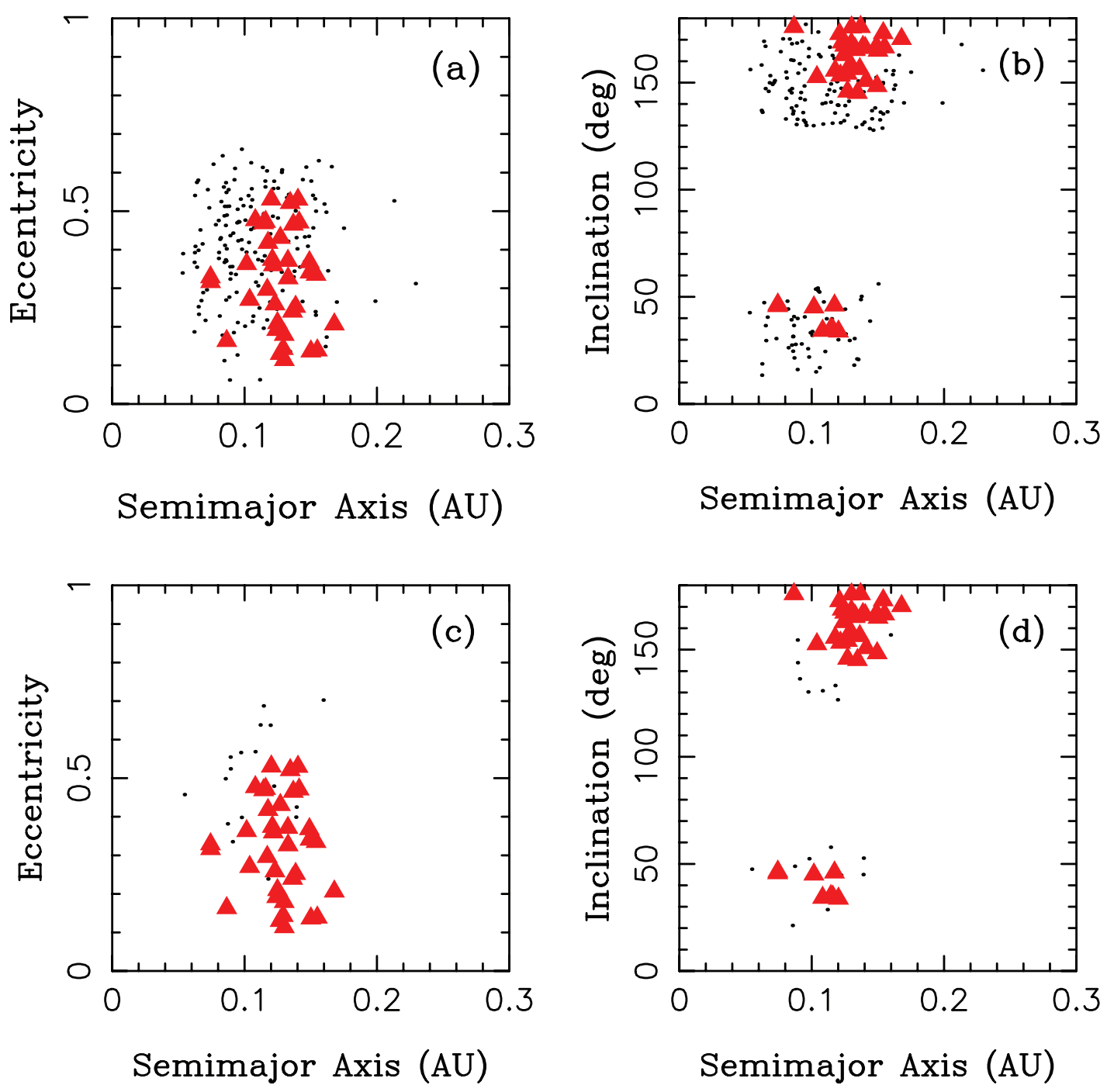

Fig. 6.-Comparison between the orbits of objects captured at Saturn in jobs $(a, b) 7$ and $(c, d) 47$ (dots) and those of the known irregular satellites at Saturn (triangles). In total, 166 and 18 stable satellites were captured in jobs 7 and 47 at Saturn, respectively.

to allow for reasonable comparison (jobs 6, 7, 9, 11, 14, 26, 30, and 47; see Table 1).

Taken together, we find that our simulations can create satellites in distant orbits at Saturn, Uranus, and Neptune with distributions that are broadly similar to the observed ones. Because Jupiter does not generally participate in planetary encounters in the Nice model ( Tsiganis et al. 2005; Gomes et al. 2005), the proposed mechanism is not expected to produce irregular satellites at Jupiter. We noted, however, that Jupiter interacted with the other planets in one migration job (run 9), and that many satellites have been produced at Jupiter in this case, 341 in total ( Table 1).

Figure 7 shows the orbits of captured objects at Jupiter (Figs. $7 a$ and $7 b$ ) and Saturn (Figs. $7 c$ and $7 d$ ) in job 9. The similarity between model and real orbits is striking except for the inclination distribution of the retrograde satellites. Also, here, as in the case of Uranus described above, the captured orbits have slightly lower inclination values than those of the real satellites' orbits. Apart from this slight discrepancy, which might indicate that the real retrograde satellites have been captured from a dynamically colder planetesimal disk than the one considered here, the agreement documented in Figure 7 is remarkable and shows that our model could potentially work for Jupiter as well. We discuss this issue in more detail in $\S 4$. Note that most retrograde satellites at Jupiter are fragments of several parent satellites that have been disrupted by collisions (Nesvorný et al. 2004). This makes a precise comparison of orbit distributions difficult.

\subsection{Satellite Generations}

Individual planetary encounters may capture, modify, and deplete satellite populations. We call the population of satellites captured in an individual encounter a "satellite generation" in what follows. Figure 8 illustrates how various satellite generations contribute to the final population of satellites at Uranus and Neptune. It shows that several dozen late generations contribute most to the final population of satellites produced at Neptune. This is because there is less opportunity to remove satellites that are captured late in the sequence of encounters.

The result for Uranus is slightly different, showing that the final population of satellites is a more complex mix of various generations (Fig. 8b). This finding is general for most of our migration runs. The contribution of various generations to the final population of satellites at Jupiter and Saturn is similar to the case of Uranus.

The planetesimal disk becomes progressively more excited in time from early to late generations of encounters. Therefore, the 


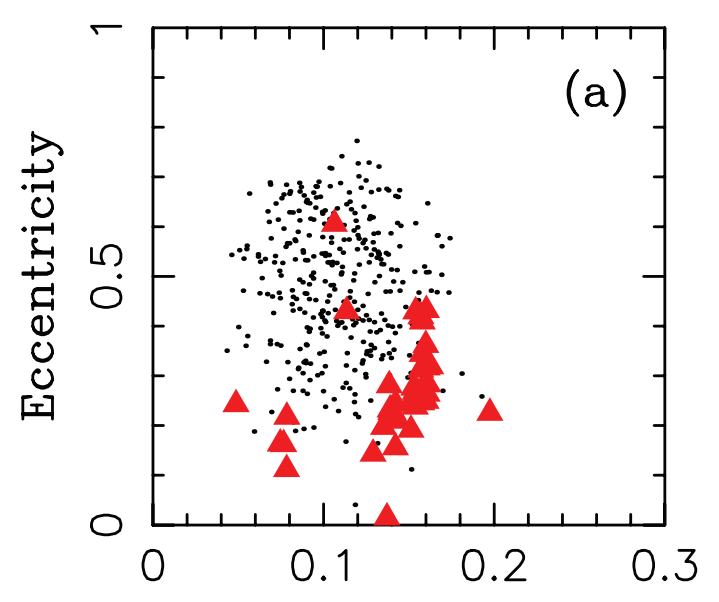

Semimajor Axis (AU)

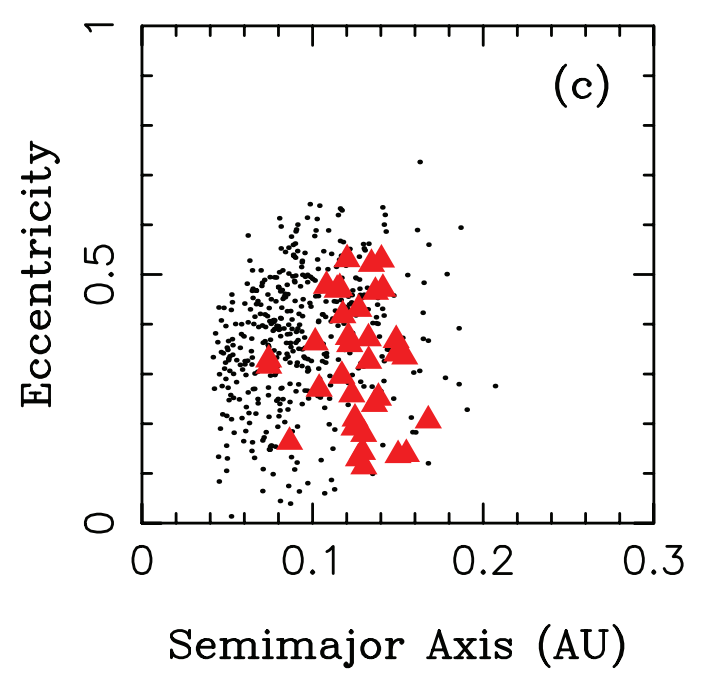

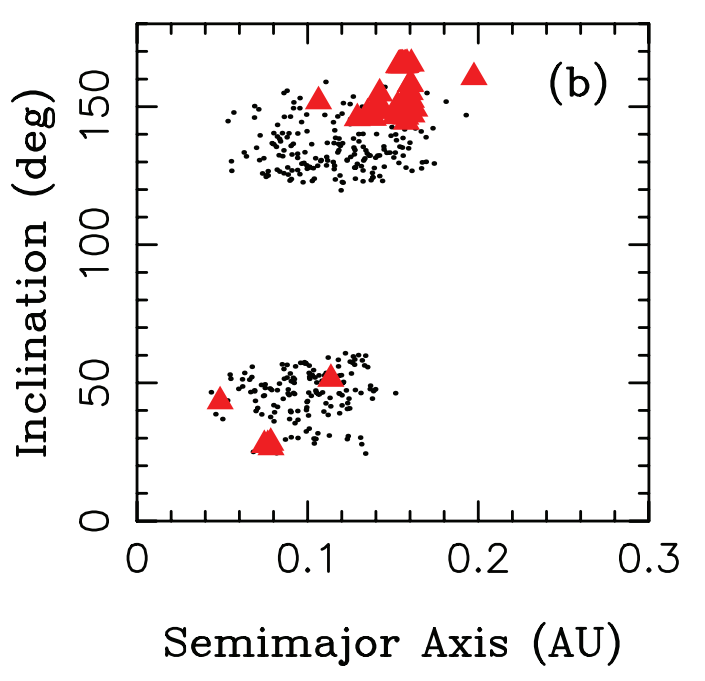

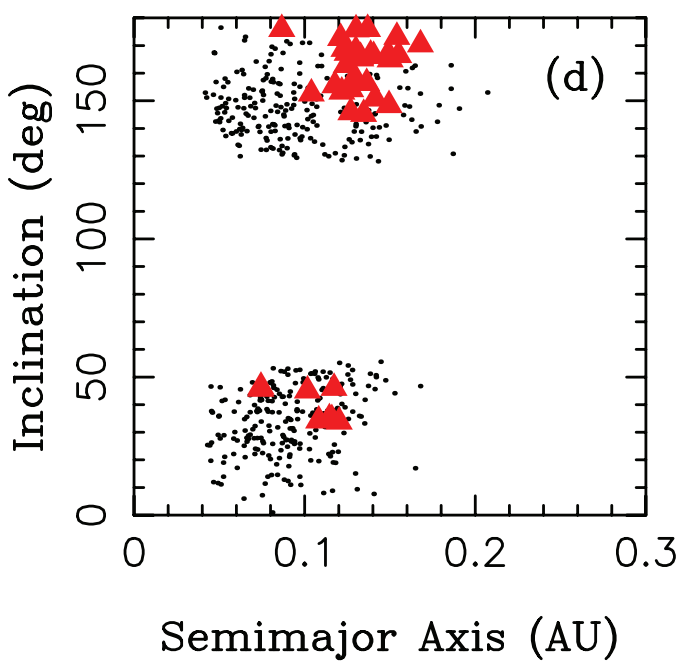

FIG. 7.-Comparison between the orbits of objects captured in job 9 (dots) and those of the known irregular satellites (triangles). $(a, b)$ Satellites of Jupiter. $(c, d)$ Satellites of Saturn. In total, 341 and 437 stable satellites were captured in job 9 at Jupiter and Saturn, respectively.

late generations of satellites, which contribute most to the final count, are captured from an excited disk and show the above mentioned $\sin i$ distribution of inclinations. This is related to the slight discrepancy between the inclination distributions of real retrograde satellites, which seem to be more clustered toward $180^{\circ}$, and the captured objects.

One way to circumvent this problem would be to somehow increase the contribution of early satellite generations to the final population of satellites, perhaps by changing the initial setup of the Nice model. We discuss this option in $\S 4$. Alternatively, the distribution of captured objects may have been shaped more aggressively (e.g., by collisions or instabilities produced by the Kozai resonance) than we determined in $\S 3.3$.

\subsection{Capture Efficiency}

Based on the results of our simulations, we can estimate the total number of satellites that we expect to have been captured by planetary encounters at each planet. We assume that the total initial mass in the planetesimal disk was $\approx 35 M_{\bullet}$. Significantly lower mass planetesimal disks cannot efficiently stabilize the scattered planets and/or cannot migrate Saturn to $9.54 \mathrm{AU}$ in 4.5 Gyr. Significantly higher mass disks would produce excessive migrations of planets and/or lower planetary $e$ and $i$ than the real ones (Tsiganis et al. 2005). The density of planetesimals is set to $1.5 \mathrm{~g} \mathrm{~cm}^{-3}$.

We will assume that the size-frequency distribution (SFD) of planetesimals at the time of planetary encounters was similar to that observed in the Kuiper Belt today. This assumption is probably correct because the SFD of large objects in the outer solar system has not changed much by collisional fragmentation since the LHB (e.g., Davis \& Farinella 2002; Kenyon \& Bromley 2004; Pan \& Sari 2005). Therefore, the current SFD of the current Kuiper Belt may be a good proxy for the SFD of planetesimals some $\sim 4$ Gyr ago.

The current SFD of classical Kuiper Belt objects can be approximated by two power laws, one for large bodies with differential power index $q_{1} \approx 4-4.5$ (Gladman et al. 2001a; Trujillo et al. 2001) and one for small bodies with differential power in$\operatorname{dex} q_{2} \approx 2-3$ (Bernstein et al. 2004; Petit et al. 2006). The above value of $q_{2}$ inferred from Kuiper Belt surveys is broadly similar to that of the ecliptic comets, which have $q \approx 2.75$ (Lamy et al. 2004). The SFD slope of small scattered disk objects is probably also similar to that of the classical Kuiper Belt (Bernstein et al. 2004).

The break in the SFD slope, $D_{\text {break }}$, occurs at diameter $D \approx$ $100 \mathrm{~km}$ (Bernstein et al. 2004) or at somewhat smaller $D$ (Petit et al. 2006). We will assume that $q_{1}=4.25, q_{2}=2.75$, and 


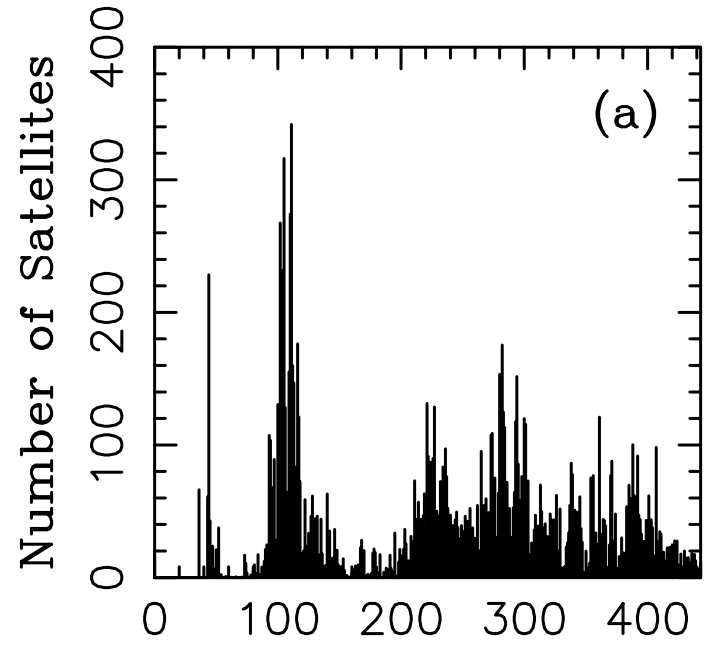

Satellite Generation

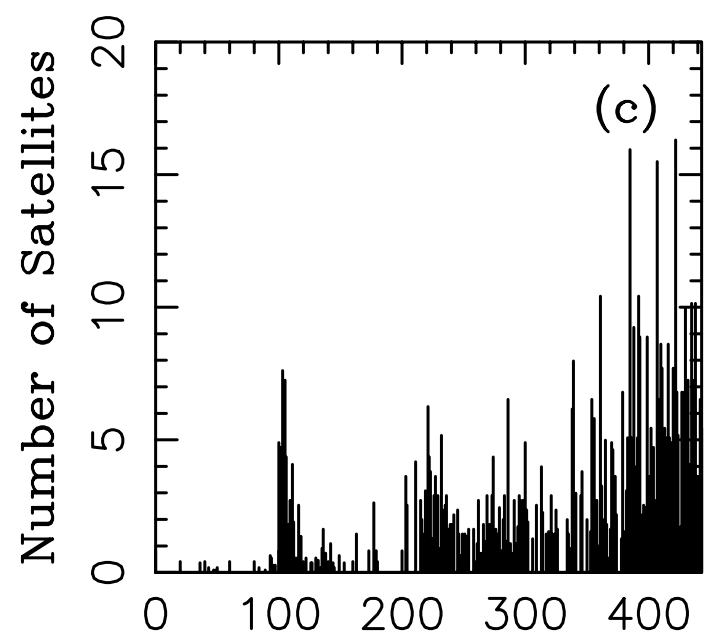

Satellite Generation



Satellite Generation

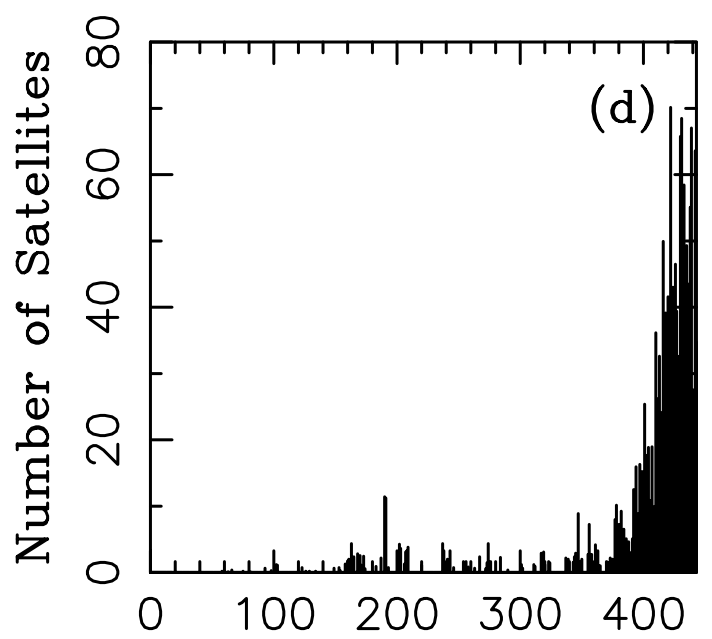

Satellite Generation

FIG. 8. - Number of satellites produced by individual planetary encounters in job 47. ( $a, c)$ Satellites of Uranus. $(b, d)$ Satellites of Neptune. The histograms in panels $a$ and $b$ do not account for satellite removal. The histograms shown in panels $c$ and $d$ account for the satellite removal during subsequent encounters and by long-term dynamical instabilities. A total of 443 planetary encounters occurred in job 47 . They are shown here in their time sequence from left to right on the abscissa. In total, Uranus and Neptune acquired 568 and 1368 stable satellites, respectively. Their orbits are shown in Fig. 5.

$D_{\text {break }}=100 \mathrm{~km}$ in the following. ${ }^{9}$ Therefore, our model SFD of the planetesimal disk is a scaled-up (to $35 M_{\bullet}$ ) version of the SFD inferred for the current Kuiper Belt. Most of the disk mass is in $\sim 10-300 \mathrm{~km}$ objects for this SFD, which is convenient because it maximizes the number of disk planetesimals in the size range of the observed irregular satellites.

From our simulations we estimate the capture probability per one particle in the disk as

$$
P_{\text {capture }}=\sum_{j=1}^{N_{\text {enc }}}\left(\frac{N_{\text {sat }, j}}{N_{\text {test }}}\right)\left(\frac{N_{\text {active }, j}}{N_{\text {total }}}\right)
$$

where index $j$ goes over individual planetary encounters recorded in a migration simulation, $N_{\text {enc }}$ is the total number of recorded encounters, $N_{\mathrm{sat}, j}$ is the number of stable satellites produced by

\footnotetext{
${ }^{9}$ We verified that the results obtained with $D_{\text {break }} \approx 25-50 \mathrm{~km}$ and/or different plausible values of $q_{1}$ and $q_{2}$ were similar to the nominal case.
}

encounter $j, N_{\text {test }}=3 \times 10^{6}$ is the number of test particles injected into the $3 \mathrm{AU}$ radius encounter sphere, $N_{\text {active, } j}$ is the number of original active particles in the encounter sphere, and $N_{\text {total }}=6868$ is the initial total number of disk particles in our migration jobs.

For Uranus and Neptune, the mean values of $P_{\text {capture }}$ over all 14 successful migration simulations are $2.6 \times 10^{-7}$ and $5.4 \times 10^{-7}$, respectively. We will assume that these mean values are representative for the capture efficiency of ice giants. For Jupiter and Saturn, for which the $P_{\text {capture }}$ strongly varies between individual jobs, it is not representative to use the mean value. As the reference values for the gas giants, we will therefore use $P_{\text {capture }}$ from job 9, for which the number of captured satellites at Jupiter and Saturn was the largest. ${ }^{10}$ These values are $8.5 \times 10^{-9}$ and $2.4 \times 10^{-8}$ for Jupiter and Saturn, respectively, and are 1 to 2 orders of magnitude lower than those of Uranus and Neptune because $N_{\mathrm{sat}, j}$ and

${ }^{10}$ We stress that job 9 serves us more as a reference rather than as a typical result. The mean number of captured satellites for Saturn over 14 migration simulations is 5.7 times smaller than the one in job 9 . 


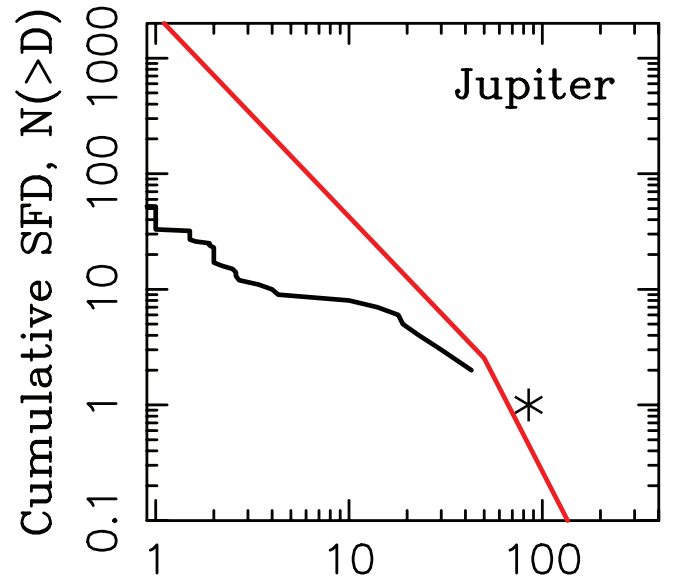

Physical Radius $(\mathrm{km})$

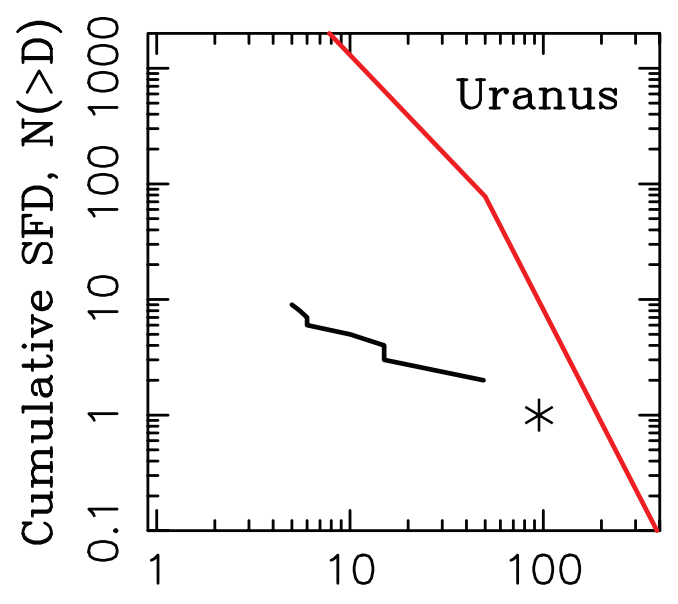

Physical Radius $(\mathrm{km})$
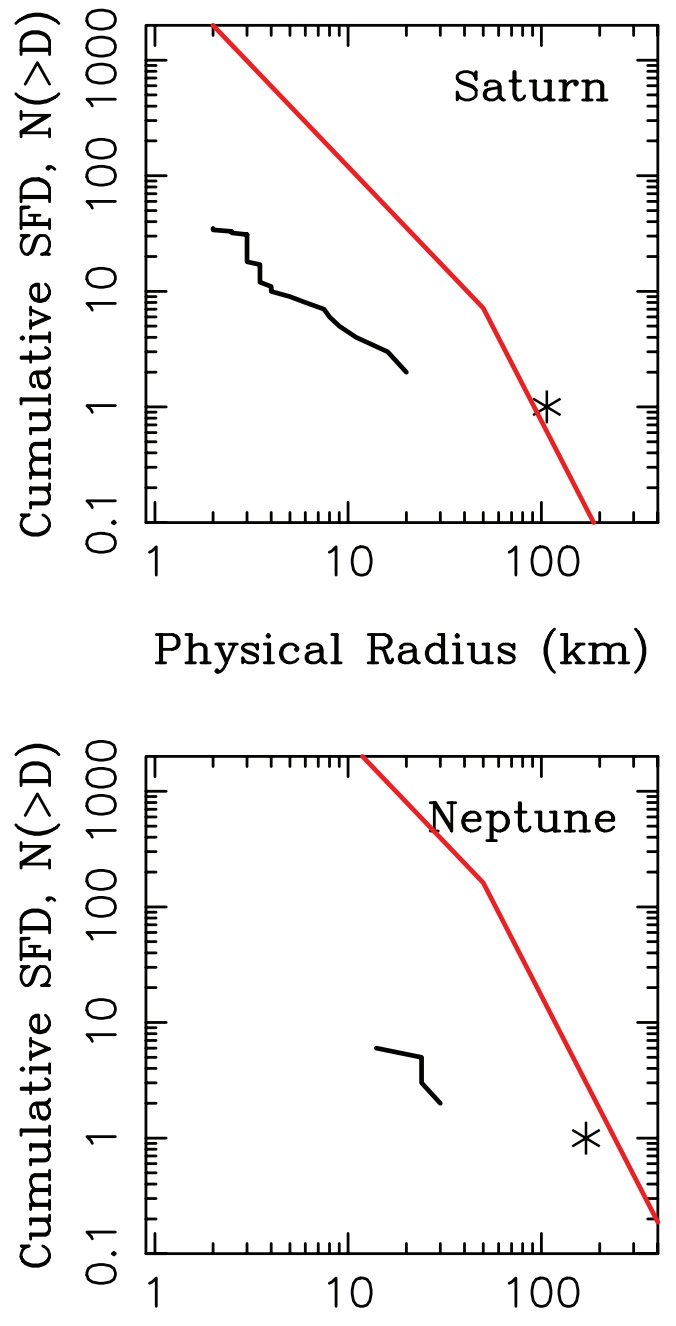

Physical Radius $(\mathrm{km})$

FIG. 9.-Comparison between SFDs of known irregular satellites (black lines; the largest moon is denoted by an asterisk) and populations of objects captured around planets in our model (red lines). To produce model populations we assumed that the planetesimal disk had the initial mass equal to $35 M_{\bullet}$ and that the SFD of disk planetesimals was represented by a broken power law similar to that given by Bernstein et al. (2004) for the current Kuiper Belt. For Jupiter and Saturn we assumed optimal capture efficiencies that occur infrequently in our simulations of the standard Nice model.

$N_{\text {active, } j}$ are relatively small for the relevant encounters. As for the difference between DE and MA classes, the DE class tends to produce larger $P_{\text {capture }}$ for Saturn. Both classes produce similar $P_{\text {capture }}$ for Uranus and Neptune.

With the assumed SFD of the planetesimal disk and the above values of $P_{\text {capture }}$, we are now able to estimate the number of satellites produced by planetary encounters at each planet. Figure 9 compares the predicted SFD of irregular satellites with the actual one.

This comparison shows that the planetary encounters are capable of producing many more irregular satellites around Uranus and Neptune than the number of currently known irregular moons at these planets. This is encouraging and leaves wiggle room for (1) a size-frequency distribution of objects in the planetesimal disk such that more of the mass was in very small or very large bodies than in the current Kuiper belt, (2) depletion of satellite populations by collisional fragmentation (Nesvorný et al. 2003, 2004), (3) dynamical depletion of irregular satellites at Neptune by captured Triton (Ćuk \& Gladman 2005), and/or (4) observational incompleteness.

According to Jewitt \& Sheppard (2005), the known populations of irregular satellites are nearly complete in their magnitude range. For example, all discovered satellites at Neptune have limiting red magnitude $\leq 25.5$, where the current detection efficiency is $>80 \%$ (Sheppard et al. 2006). Moreover, the dynamical depletion due to Triton is specific to Neptune and does not apply to other planets (while the excess of small satellites captured by planetary encounters applies also to Uranus and may be common to all planets). Therefore, we believe that (3) and (4) do not apply. We discuss (1) and (2) in $\S 4$.

For Jupiter and Saturn, for which we used $P_{\text {capture }}$ for a selected (optimal) migration scenario (job 9), the number of captured small satellites is also significantly larger than the number of small irregular satellites known at these planets (Fig. 9). Moreover, the number of large captured objects at these planets is sufficient (with our optimistic values of $P_{\text {capture }}$ ) to explain the existence of the largest known irregular moons (Himalia at Jupiter with $D \approx 170 \mathrm{~km}$ and Phoebe at Saturn with $D \approx 213 \mathrm{~km})$. In fact, the largest irregular satellites captured by planetary encounters at Jupiter and Saturn have $D \approx 135$ and $186 \mathrm{~km}$ and are only slightly smaller than Himalia and Phoebe, respectively. On surface, this result may be viewed as another success of our model. The infrequent occurrence of migration jobs like those of 
run 9 probably indicates, however, that we are still missing some important element in our understanding of how exactly the irregular satellites of Jupiter and Saturn formed. As we discuss below, this problem may be rooted in the precise ways the outer planets formed in and interacted with the planetesimal disk.

\section{DISCUSSION}

We showed above that the formation of distant satellites at outer planets is a natural by-product of the Nice model (Tsiganis et al. 2005; Gomes et al. 2005). The Nice model postulates that the four outer planets formed in a compact configuration between 5 and $\approx 20 \mathrm{AU}$, scattered from each other, and migrated in the planetesimal disk to their current radial locations. The irregular satellites are captured in the Nice model by three-body gravitational reactions during a planetary encounters, when pairs of planets deflect objects from the background planetesimal disk into stable planet-bound orbits. We showed that the orbits of satellites captured by this process are broadly similar to those of the known irregular moons.

In the Nice model, as originally proposed by Tsiganis et al. (2005), Uranus and Neptune encounter each other many times, which assures that (1) any populations of distant satellites captured prior to the epoch of planetary encounters at these planets are removed or severely depleted, and (2) there are many opportunities for captures by three-body reactions of new satellites from the background planetesimal disk. As a consequence of (2), the capture efficiency of satellites at Uranus and Neptune is large. If it is assumed that the shape of the SFD of disk planetesimals was comparable to that inferred from observations of today's Kuiper Belt and that the total disk mass between 20 and 35 AU was $\sim 35 M_{\bullet}$, we estimate that the population of small irregular satellites produced by planetary encounters at Uranus and Neptune may have been more than 2 orders of magnitude larger than the number of currently known irregular satellites at these planets ( $§ 3.5$ ). This result is puzzling.

In fact, one common characteristic of known irregular moons is that their SFDs are very shallow for $D \gtrsim 10 \mathrm{~km}$ (differential power index $\approx 2) .{ }^{11}$ This very shallow SFD is difficult to explain unless we assume that the SFD of disk planetesimals was similarly shallow between 10 and $\sim 300 \mathrm{~km}$ diameters at the time when the satellites were captured. ${ }^{12}$

A shallow SFD of the planetesimal disk could have been produced as a result of the accretion process (Stern \& Colwell 1997; Kenyon \& Bromley 2004) and would have been maintained only if the disk suffered limited erosion via collisional fragmentation. Therefore, the SFD of irregular satellites may be a fossil remnant of the SFD at the epoch of planetary encounters in the Nice model. If these conjectures are correct, the results discussed here would place important constraints on the state of the planetesimal disk at the time of planetary encounters.

The fragmentation evolution of a planetesimal disk has a minor effect on its SFD either if the disk stays dynamically cold or if

\footnotetext{
11 For Jupiter and Saturn, for which there are enough statistics to infer the SFD for $D<10 \mathrm{~km}$, there is an indication that the shallow SFD for $D \gtrsim 10 \mathrm{~km}$ steepens for $D<10 \mathrm{~km}$, possibly because of numerous fragments produced by collisions in this size range (Nesvorný et al. 2004). The SFD of irregular satellites at Neptune is not well determined given the few objects known to date (Sheppard et al. 2006).

${ }^{12}$ An interesting and possibly related fact is that the SFD of long-periodic comets is probably very shallow as well (Weissman 1996). This could indicate that the irregular satellites and Oort cloud comets collisionally decoupled from the planetesimal disk before its SFD developed a steeper slope, such as the one today characterizing Jupiter's Trojans, the ecliptic comets, the scattered disk, and the classical Kuiper belt.
}

the relevant time interval is short. In the latter case, the capture of irregular satellites would have to occur relatively early. This, in turn, could rule out the LHB variant of the Nice model in which the planetary encounters occur $\sim 800 \mathrm{Myr}$ after the formation of the solar system. Alternatively, the planetesimal disk would stay dynamically cold over $\sim 800$ Myr only if the accretion was extremely slow and did not produce excessively large planetesimals that would gravitationally perturb the disk. This possibility, however, could be difficult to reconcile with the current Kuiper Belt, in which Pluto and several Pluto-size objects are known to exist (e.g., Brown 2005). Therefore, we believe that it is rather unlikely that the SFD of planetesimals was shallow at the time when irregular satellites were captured.

A different and probably more plausible explanation for the shallow SFD of the irregular satellites with $D=10-300 \mathrm{~km}$ can be invoked if their SFD evolved over time to a shallower slope by collisional disruptions of moons with $D=10-300 \mathrm{~km}$ (item 2 in $\S 3.5$ ). This possibility would imply that most captured $D=10-300 \mathrm{~km}$ irregular satellites were disrupted, with a progressively larger disruption efficiency toward smaller diameters, producing fragments with $D<10 \mathrm{~km}$. The observed steeper slope of the SFD of the irregular satellites at Jupiter and Saturn for $D<10 \mathrm{~km}$ may hint at such a process. Conversely, only a very few fragments with $D>10 \mathrm{~km}$ could have been produced by collisional disruptions because of the lack of parent irregular satellites large enough to produce these large fragments (Fig. 9).

Two possibilities exist: (1) disruptions by collisions between irregular moons, and (2) collisional disruptions produced by the LHB planetesimals that penetrate in large quantities into planet's Hill spheres and impact the existing irregular satellites. As for (1), the large populations of irregular moons produced by the planetary encounters must undergo important changes via mutual collisions between irregular moons over $\sim 4$ Gyr (Nesvorný et al. 2003). The evolution of the SFD produced by such mutual collisions can be investigated via simulations of the collisional cascade with the currently available codes (e.g., Bottke et al. 2005).

As for (2), Nesvorný et al. (2004) have estimated the rate of collisional disruptions in the standard Malhotra model for smooth migration of planets (Malhotra 1995; Hahn \& Malhotra 1999). (This model should not be confused with the MA class of migration in Nice model described in $\S 3.1$; e.g., the outer planets start and remain on near-circular orbits in the standard Malhotra model.) They showed that the disruption rate of irregular moons depends sensitively on the assumed SFD of planetesimals. Their results, which may fit best into the framework discussed here, are the ones for which the steep SFD of planetesimals (power index $q=4.2$ ) continues down to $\approx 10 \mathrm{~km}$ diameter (distribution $N_{C}$ in Nesvorný et al. 2004). In such a case, the irregular satellites with $D<100 \mathrm{~km}$ become severely depleted by collisions. A model with coupled orbital and collisional evolution of irregular satellites and disk planetesimals in the Nice model will be needed to address this issue in more detail.

Our model capture efficiencies for Saturn and especially for Jupiter are significantly lower than those for Uranus and Neptune. This would suggest that Saturn and Jupiter should have acquired populations of irregular satellites that are significantly less numerous than the ones at Uranus and Neptune. This directly contradicts the results of Jewitt \& Sheppard (2005), who inferred from observations that all outer planets have similar populations of irregular satellites. Therefore, (1) the original Nice model should be modified to accommodate more planetary encounters involving Jupiter and Saturn, or (2) the irregular satellites at Jupiter and Saturn formed by a different mechanism than the one advocated here for those at Uranus and Neptune. With (2), the population 
similarity pointed out by Jewitt \& Sheppard (2005) would be a mere coincidence. We discuss cases (1) and (2) below.

As for (2), there exists some evidence that Jupiter's irregular satellites may have formed early and by a different mechanism than the one discussed here. For example, Pasiphae and Sinope, which are the two largest retrograde irregular moons at Jupiter, have resonant orbits (Saha \& Tremaine 1993; Whipple \& Shelus 1993; Nesvorný et al. 2003; Beaugé \& Nesvorný 2007). These resonant orbits could have been produced by a gradual orbit decay of large moons produced by gas drag. (Note that the gradient of secular frequencies of irregular moons with $a$ is large so that any changes in planetary frequencies [perhaps due to planet migration or depletion of the planetesimal disk] cannot significantly sweep resonances in the orbit space.) This, in turn, would require an early formation of Jupiter's irregular moons and their survival over the epoch of planetary encounters in the Nice model. Note that at least two of Saturn's irregular satellites also have resonant orbits, while all known irregular satellites at Uranus and Neptune have nonresonant orbits (see, e.g., Beaugé \& Nesvorný 2007).

Option 2 is plausible in the original Nice model (Tsiganis et al. 2005; Gomes et al. 2005), in which Jupiter participates in planetary encounters only in a very limited number of migration cases. Consequently, the formation of Jupiter's irregular satellites by planetary encounters in the original Nice model is unlikely. Current research indicates, however, that the outer planets may have started in an even more compact configuration than the one considered in the original Nice model. Specifically, Jupiter and Saturn may have been driven by planet-gas interactions into their mutual 2:3 mean motion resonance (Morbidelli et al. 2005a). Moreover, the orbits of Uranus and Neptune might have been placed in similar mean motion resonances as well.
Our preliminary migration simulations that started from these tight resonant configurations of the outer planets produce many more planetary encounters of Jupiter and Saturn than those in the original Nice model. This is an encouraging result, which could suggest that the more realistic starting conditions could lead to more equal distributions of planetary encounters among the outer planets and consequently to more similar populations of captured objects. Such a result would be easier to reconcile with the similar numbers of irregular satellites present at each outer planet (Jewitt \& Sheppard 2005).

Our new model may provide a plausible explanation for the origin of Neptune's large moon Triton. As we discussed above, most objects captured during planetary encounters have unstable orbits with very large values of $e$. We have not considered these orbits in our work. The initially eccentric orbit of a Triton-sized object could have been efficiently stabilized, however, by dissipative effects (see Nicholson et al. 2007 for a recent review). Therefore, the numerous orbits with high $e$ captured during planetary encounters in our model may represent possible analogs for Triton's orbit before it was stabilized by dissipative effects. Further work will be needed to estimate the efficiency of this capture mechanism.

We thank Rodney Gomes for giving us the initial conditions for the migration jobs. We thank Hal Levison, Luke Dones, Bill Bottke, and Cristian Beaugé for motivating discussions. The support for the work of D. N. was provided by the National Science Foundation. The work of D. V. was partially supported by the Czech Grant Agency through grant 205/05/2737.
Agnor, C. B., \& Hamilton, D. P. 2006, Nature, 441, 192

Beaugé, C., \& Nesvorný, D. 2007, AJ, in press

Beaugé, C., Roig, F., \& Nesvorný, D. 2002, Icarus, 158, 483

Bernstein, G. M., Trilling, D. E., Allen, R. L., Brown, M. E., Holman, M., \& Malhotra, R. 2004, AJ, 128, 1364

Bottke, W. F., Durda, D., Nesvorný, D., Jedicke, R., Morbidelli, A., \& Levison, H. 2005, Icarus, 175, 111

Brown, M. E. 2000, AJ, 119, 977

Brown, M. E., Trujillo, C. A., \& Rabinowitz, D. L. 2005, ApJ, 635, L97

Canup, R. M., \& Ward, W. R. 2002, AJ, 124, 3404 2006, Nature, 441, 834

Carruba, V., Burns, J. A., Nicholson, P. D., \& Gladman, B. J. 2002, Icarus, 158, 434

Colombo, G., \& Franklin, F. A. 1971, Icarus, 15, 186

Cruikshank, D. P. 1980, Icarus, 41, 246

Cuk, M., \& Burns, J. A. 2004, Icarus, 167, 369

Ćuk, M., \& Gladman, B. J. 2005, ApJ, 626, L113 2006, Icarus, 183, 362

Davis, D. R., \& Farinella, P. 2002, Highlights Astron., 12, 219

Degewij, J., Cruikshank, D. P., \& Hartmann, W. K. 1980, Icarus, 44, 541

Dones, L., Weissman, P. R., Levison, H. F., \& Duncan, M. J. 2004, in Comets II, ed. M. C. Festou, H. U. Keller, \& H. A. Weaver (Tucson: Univ. Arizona Press), 153

Dumas, C., Owen, T., \& Barucci, M. A. 1998, Icarus, 133, 221

Duncan, M. J., Levison, H. F., \& Lee, M. H. 1998, AJ, 116, 2067

Fernandez, J. A., \& Ip, W. H. 1984, Icarus, 58, 109

Gladman, B., Kavelaars, J. J., Holman, M., Petit, J.-M., Scholl, H., Nicholson, P. D., \& Burns, J. A. 2000, Icarus, 147, 320

Gladman, B., Kavelaars, J. J., Petit, J.-M., Morbidelli, A., Holman, M. J., \& Loredo, T. 2001a, AJ, 122, 1051

Gladman, B. J., Nicholson, P. D., Burns, J. A., Kavelaars, J. J., Marsden, B. G., Williams, G. V., \& Offutt, W. B. 1998, Nature, 392, 897

Gladman, B., et al. 2001b, Nature, 412, 163

Goldreich, P., \& Tremaine, S. 1979, ApJ, 233, 857 1980, ApJ, 241, 425

Gomes, R. S. 1998, AJ, 116, 2590

Gomes, R., Levison, H. F., Tsiganis, K., \& Morbidelli, A. 2005, Nature, 435, 466

\section{REFERENCES}

Gomes, R. S., Morbidelli, A., \& Levison, H. F. 2004, Icarus, 170, 492

Grav, T., \& Holman, M. J. 2004, ApJ, 605, L141

Grav, T., Holman, M. J., \& Fraser, W. C. 2004, ApJ, 613, L77

Grav, T., Holman, M. J., Gladman, B. J., \& Aksnes, K. 2003, Icarus, 166, 33

Greenberg, R., Weidenshilling, S. J., Chapman, C. R., \& Davis, D. R. 1984, Icarus, 59, 87

Hahn, J. M., \& Malhotra, R. 1999, AJ, 117, 3041

Hartmann, W. K., Ryder, G., Dones, L., \& Grinspoon, D. 2000, in Origin of the Earth and Moon, ed. R. M. Canup et al. (Tucson: Univ. Arizona Press), 493 Heppenheimer, T. A., \& Porco, C. 1977, Icarus, 30, 385

Holman, M. J., et al. 2004, Nature, 430, 865

Hut, P., \& Bahcall, J. N. 1983, ApJ, 268, 319

Jewitt, D., \& Luu, J. 1993, Nature, 362, 730

Jewitt, D., \& Sheppard, S. 2005, Space Sci. Rev., 116, 441

Kaula, W. M., \& Yoder, C. F. 1976, Lunar Planet. Sci. Conf., 7, 440

Kavelaars, J. J., et al. 2004, Icarus, 169, 474

Kenyon, S. J. 2002, PASP, 114, 265

Kenyon, S. J., \& Bromley, B. C. 2004, AJ, 128, 1916

Kortenkamp, S. J. 2005, Icarus, 175, 409

Kozai, Y. 1962, AJ, 67, 591

Lamy, P. L., Toth, I., Fernandez, Y. R., \& Weaver, H. A. 2004, in Comets II, ed. M. C. Festou, H. U. Keller, \& H. A. Weaver (Tucson: Univ. Arizona Press), 223

Levison, H. F., Dones, L., Chapman, C. R., Stern, S. A., Duncan, M. J., \& Zahnle, K. 2001, Icarus, 151, 286

Levison, H. F., \& Morbidelli, A. 2003, Nature, 426, 419

Liou, J. C., \& Malhotra, R. 1997, Science, 275, 375

Lubow, S. H., Seibert, M., \& Artymowicz, P. 1999, ApJ, 526, 1001

Luu, J. X., \& Jewitt, D. C. 2002, ARA\&A, 40, 63

Malhotra, R. 1995, AJ, 110, 420

Michel, P., Benz, W., Tanga, P., \& Richardson, D. C. 2001, Science, 294, 1696 Morbidelli, A., Crida, A., \& Masset, F. 2005a, BAAS, 37, 667

Morbidelli, A., Levison, H. F., Tsiganis, K., \& Gomes, R. 2005b, Nature, 435, 462

Mosqueira, I., \& Estrada, P. R. 2003, Icarus, 163, 198

Nesvorný, D., Alvarellos, J. L. A., Dones, L., \& Levison, H. F. 2003, AJ, 126, 398

Nesvorný, D., Beaugé, C., \& Dones, L. 2004, AJ, 127, 1768 
Nicholson, P. D., Ćuk, M., Sheppard, S. S., Nesvorný, D., \& Johnson, T. V. 2007, in Kuiper Belt, ed. A. Barucci et al. (Tucson: Univ. Arizona Press), in press

Pan, M., \& Sari, R. 2005, Icarus, 173, 342

Petit, J.-M., Holman, M. J., Gladman, B. J., Kavelaars, J. J., Scholl, H., \& Loredo, T. J. 2006, MNRAS, 365, 429

Petit, J.-M., Morbidelli, A., \& Chambers, J. 2001, Icarus, 153, 338

Pollack, J. B., Burns, J. A., \& Tauber, M. E. 1979, Icarus, 37, 587

Pollack, J. B., Haberle, R. M., Schaeffer, J., \& Lee, H. 1991, BAAS, 23, 1214

Pollack, J. B., Hubickyj, O., Bodenheimer, P., Lissauer, J. J., Podolak, M., \& Greenzweig, Y. 1996, Icarus, 124, 62

Porco, C. C., et al. 2005, Science, 307, 1237

Press, W. H., Teukolsky, S. A., Vetterling, W. T., \& Flannery, B. P. 1992, Numerical Recipes in FORTRAN (2nd ed; Cambridge: Cambridge Univ. Press)

Safronov, V. S. 1991, Icarus, 94, 260

Saha, P., \& Tremaine, S. 1993, Icarus, 106, 549

Sheppard, S. S., Gladman, B., \& Marsden, B. G. 2003, IAU Circ. 8116, 1

Sheppard, S. S., \& Jewitt, D. C. 2002, BAAS, 34, 881
Sheppard, S. S., \& Jewitt, D. C. 2003, Nature, 423, 261

Sheppard, S. S., Jewitt, D., \& Kleyna, J. 2005, AJ, 129, 518 . 2006, AJ, 132, 171

Skeel, R. D., \& Biesiadecki, J. J. 1994, Ann. Num. Math., 1, 1

Stern, S. A., \& Colwell, J. E. 1997, AJ, 114, 841

Stevenson, D. 2001, Science, 294, 71

Sykes, M. V., Nelson, B., Cutri, R. M., Kirkpatrick, D. J., Hurt, R., \& Skrutskie, M. F. 2000, Icarus, 143, 371

Touma, J., \& Wisdom, J. 1998, AJ, 115, 1653

Trujillo, C. A., Jewitt, D. C., \& Luu, J. X. 2001, AJ, 122, 457

Tsiganis, K., Gomes, R., Morbidelli, A., \& Levison, H. F. 2005, Nature, 435, 459

Weissman, P. R., \& Levison, H. 1996, Lunar Planet. Sci., 27, 1409

Wetherill, G. W. 1989, in Asteroids II, ed. R. P. Binzel et al. (Tucson: Univ. Arizona Press), 661 . 1992, Icarus, 100, 307

Whipple, A. L., \& Shelus, P. J. 1993, Icarus, 101, 265

Wisdom, J., \& Holman, M. 1991, AJ, 102, 1528 